\title{
Nanoparticle-induced vascular blockade in human prostate cancer
}

Lilach Agemy ${ }^{1}$, Kazuki N. Sugahara ${ }^{1}$, Venkata Ramana Kotamraju ${ }^{1}$, Kunal Gujraty ${ }^{1}$, Olivier M. Girard $^{2}$, Yuko Kono², Robert F. Mattrey², Ji-Ho Park ${ }^{3}$, Michael J. Sailor ${ }^{3}$, Ana I. Jimenez ${ }^{4}$, Carlos Cativiela $^{4}$, David Zanuy ${ }^{5}$, Francisco J. Sayago ${ }^{4}$, Carlos Aleman ${ }^{5}$, Ruth Nussinov ${ }^{6,7}$ and Erkki Ruoslahti ${ }^{1,8 *}$

${ }^{1}$ Vascular Mapping Laboratory, Center for Nanomedicine, Sanford-Burnham Medical Research Institute at UCSB, 3119 Biology II Bldg., University of California, Santa Barbara, CA 93106-9610

${ }^{2}$ Department of Radiology, University of California, San Diego, CA 92103

${ }^{3}$ Materials Science and Engineering Program and Department of Chemistry and Biochemistry, University of California, San Diego, 9500 Gilman, La Jolla, CA 92093

${ }^{4}$ Organic Chemistry Department, ICMA, University of Zaragoza-CSIC, 50009 Zaragoza, Spain ${ }^{5}$ Department of Chemical Engineering, ETSEIB, Polytechnic University of Catalonia, Diagonal 647, Barcelona E-08028, Spain

${ }^{6}$ Basic Science Program, Center for Cancer Research Nanobiology Program, SAIC-Frederick, Inc., NCI, Frederick, MD 21702

${ }^{7}$ Department of Human Genetics, Sackler, Medical School, Tel Aviv University, Tel Aviv 69978, Israel

${ }^{8}$ Cancer Research Center, Sanford-Burnham Medical Research Institute, La Jolla, CA 92037

*Correspondence: ruoslahti@burnham.org, 805-893-5327 (phone), 805-893-5805 (fax).

Key words: Peptides, non-proteinogenic amino acids, tumor targeting, tumor blood vessels, blood clotting, MRI, contrast-enhanced ultrasound imaging, tumor treatment 


\section{Abstract}

The tumor-homing pentapeptide CREKA specifically homes to tumors by binding to fibrin and fibrin-associated clotted plasma proteins in tumor vessels. Previous results show that CREKAcoated superparamagnetic iron oxide particles can cause additional clotting in tumor vessels, which creates more binding sites for the peptide. We have used this self-amplifying homing system to develop theranostic nanoparticles that simultaneously serve as an imaging agent and inhibit tumor growth by obstructing tumor circulation through blood clotting. The CREKA nanoparticles were combined with nanoparticles coated with another tumor-homing peptide, CRKDKC, and nanoparticles with an elongated shape (nanoworms) were employed for improved binding efficacy. The efficacy of the CREKA peptide was then increased by replacing some residues with non-proteinogenic counterparts, which increased the stability of the peptide in the circulation. Treatment of mice bearing orthotopic human prostate cancer tumors with the targeted nanoworms caused extensive clotting in tumor vessels, whereas no clotting was observed in the vessels of normal tissues. Optical and magnetic resonance imaging confirmed tumor-specific targeting of the nanoworms, and ultrasound imaging showed reduced blood flow in tumor vessels. Treatment of prostate cancer mice with multiple doses of the nanoworms induced tumor necrosis and highly significant reduction in tumor growth. 


\section{Introduction}

The prevalence of prostate cancer and the large number of deaths from this disease underscore the need for a paradigm shift in the strategies to better treat this cancer ${ }^{1}$. Since the 1970's, progress in fundamental cancer biology has led to enormous advances in our understanding of the processes that underlie malignant transformation and metastatic dissemination. Nonetheless, eradication of cancer remains an elusive clinical goal, largely due to the heterogeneous nature of individual cancers and our inability to target therapies to neoplastic cells without damaging normal tissues. Prostate tumors are anatomically, histologically and genetically heterogeneous ${ }^{2-4}$, causing variable responses to various therapies. These obstacles are further magnified by our limited ability to image cancerous regions and track the progression of treatments ${ }^{5-6}$.

One approach to overcome the heterogeneity of tumors is to focus on tumor vasculature. Tumor vasculature has proven to be particularly well suited as a site for homing-based (synaphic) targeting. It expresses a multitude of molecules that are not expressed in the vessels of normal tissues, and the vascular wall is readily accessible for blood-borne substances ${ }^{7}$. Most vascular strategies use anti-angiogeneic therapy to prevent formation of new blood vessels in a growing solid tumor; these approaches have been found to be useful, particularly in treating advancedstage cancers ${ }^{8-12}$. An alternative strategy is based on occluding the vasculature of a tumor and thereby inducing tumor necrosis. Targeting of truncated tissue factor to tumors has been used for this purpose with some success ${ }^{13-17}$. Nanomedicine is an emerging field that uses nanoparticles to facilitate the diagnosis and treatment of diseases. Nanoparticles can be engineered to perform multiple functions, which provide a potential advantage over simple drugs. In this study we designed nanomedicine-based approaches to more effectively and safely block the tumor circulation.

Our laboratory screens phage-displayed peptide libraries in vivo and ex vivo to discover specific targets in tumor vessels ${ }^{18}$. Some of the tumor-homing peptides we have identified in this manner recognize products of blood clotting on the walls of tumor vessels and in tumor stroma 
that are not present in normal vessels and tissues. The reasons for this difference are thought to be a pro-coagulant tumor environment and seepage of plasma proteins including fibrinogen, from the leaky tumor vessels into the tissue ${ }^{19-20}$. We have identified three tumor-homing peptides that recognize these clotting products in the vessels of a variety of tumor types, including human cancers ${ }^{21-22}$. Tumors grown in mutant mice null for fibrinogen, or mice lacking plasma fibronectin, which becomes covalently bound to fibrin during blood clotting, are not recognized by these peptides, indicating that the peptides target fibrin-fibronectin complexes.

We have recently used one of the peptides that recognize fibrin-fibronectin complexes, a pentapeptide with the sequence CREKA, to design a self-amplifying nanoparticle delivery system ${ }^{22}$. Iron oxide nanoparticles coated with this peptide accumulate in tumor vessels, where they induce additional local clotting, and thereby produce new binding sites for more particles. This amplification system enhanced homing of the nanoparticles in a mouse tumor model without causing clotting or other obvious side effects elsewhere in the body. The self-amplified tumor accumulation produced enhancement of tumor imaging, but significant inhibition of tumor growth was not obtained. We set out to develop an effective theranostic system based on the earlier findings using prostate cancer as the target tumor. 


\section{Methods}

Cell lines and tumors. The 22Rv1 prostate cancer cell line was obtained from American Type Culture Collection (Manassas, VA). The cells were grown in RPMI 1640 media supplemented with $2 \mathrm{mM}$ glutamine, $1 \%$ penicillin-streptomycin, and $10 \% \mathrm{FCS}$ at $37^{\circ} \mathrm{C}$ and $5 \% \mathrm{CO}_{2}$. Xenografts were created by injecting BALB/c nude mice with $2 \times 10^{6}$ cells in PBS, orthotopically into the prostate gland. The LAPC9 human prostate adenocarcinoma xenograft was from Dr. Lily Wu (University of California, Los Angeles) and used to produce tumors as described ${ }^{23}$. Animal experimentation was performed according to procedures approved by the Animal Research Committee at the University of California, Santa Barbara, and the Sanford-Burnham Medical Research Institute, San Diego.

Peptide synthesis. Peptides were synthesized with an automatic microwave assisted peptide synthesizer (Liberty; CEM, Matthews, NC) using standard solid-phase Fmoc/t-Bu chemistry with 2-(1H-7-Azabenzotriazol-1-yl)-1,1,3,3-tetramethyl uronium hexafluorophosphate Methanaminium (Anaspec, Inc., San Jose, California, USA) as the coupling reagent. During synthesis, the peptides were labeled with 5(6)-carboxyfluorescein (FAM) (Sigma-Aldrich, St. Louis, MO USA) with a 6-aminohexanoic acid spacer separating the dye from the sequence. The peptides were cleaved from the resin using 95\% Trifluoroacetic acid (Sigma-Aldrich, St. Louis, MO, USA) with $2.5 \%$ water and triisiopropylsilane (Sigma-Aldrich, St. Louis, MO USA). Subsequent purification by High Performance Liquid Chromatography (Gilson Inc., Middleton, Wisconsin, USA) gave peptides with $>90 \%$ purity. The preparation of the non-proteinogenic amino acids used for the synthesis of the N/C $\alpha$-methylated CREKA analogs will be reported elsewhere. The synthesis of CRKDKC with an extra N-terminal cysteine used for the chemoselective ligation used in this work will be described elsewhere.

Computer modeling. The conformational profiles of the CREKA analogs, which were constructed by replacing each residue (one-by-one) by its $N$-methyl or $C \alpha$-methyl counterpart, were characterized by Molecular Dynamics simulations. Methodology and clustering analyses were as described in ${ }^{24}$.

Preparation of NW and Ni-liposomes. NW ${ }^{25}$, NW coated with peptides and Ni-liposomes were prepared as described ${ }^{22}$. The aminated nanoworms were pegylated with maleimide-5KPEG- 
NHS (JenkemTechniology, China). In the experiments where the Ni-liposomes were used, the nanoworms were functionalized for subsequent peptide coupling with $(\mathrm{N}-[\alpha-$ maleimidoacetoxy]succinimide ester (AMAS), (Pierce, Rockford, IL). Peptides were conjugated to the nanoparticles using Michael addition reaction between the thiol from a cysteine in the peptide sequence and the maleimide on the functionalized particles.

In vivo peptide homing. Orthotopic prostate cancers were used when they reached $0.5-1 \mathrm{~cm}$ in size. Synthetic peptides labeled with FAM (approximately $200 \mu \mathrm{g}$ ) were intravenously injected into tumor-bearing mice and allowed to circulate for 15 minutes to 3 hours. The mice were perfused with PBS through the heart under anesthesia, and tissues were collected and observed under UV light (Illumatool Bright Light System LT-9900, Lightools Research, Encinitas, CA), and then processed for immunofluorescence or immunohistochemistry.

In vivo NW injections. To analyze NW biodistribution, mice bearing orthotopic $22 \mathrm{Rv} 1$ tumors were injected into the tail vein ( $5 \mathrm{mg}$ of iron per $\mathrm{kg}$ body weight). In homing experiments, the mice were euthanized 5-6 hours after the injection by cardiac perfusion with PBS under anesthesia, and organs were dissected and analyzed for particle homing. In tumor treatment experiments, nude mice bearing 2 week-old 22Rv-1 or LAPC9 orthotopic xenografts (typically about 200-250 $\mathrm{mm}^{3}$ in tumor volume) were intravenously injected with NW in $150 \mu \mathrm{I}$ PBS or PBS as a control. The NW were injected every other day for 14 days. The total cumulative dose was $35 \mathrm{mg}$ iron $/ \mathrm{kg}$. At the end of the treatment, the mice were perfused with PBS under anesthesia and the tumors were harvested. Tumor volume was calculated using the following formula: volume $=\left(d^{2} \times D\right) / 2$, where $d$ is the smallest and $D$ are the largest tumor diameters ${ }^{26}$.

Peptide stability. The peptides were conjugated with a linker that bridges the NW and a cysteine residue on the peptide, forming a disulfide by reacting with the (ortho-pyridyl)disulfide group. The peptide-coated NW were injected into animals, and blood was collected 15 minutes and 3 hours after the injection. The plasma was separated and NW were collected by ultracentrifugation $\left(100,000 \times \mathrm{g}\right.$ for 10 minutes at $\left.4^{\circ} \mathrm{C}\right)$. Proteins bound to the $\mathrm{NW}$ were removed by incubation with glycine- $\mathrm{HCl}, \mathrm{pH} 2.8$ on a magnetic LS columns (MACS separation columns, Miltenyi Biotec, Bergisch Gladbach, Germany). The peptides were cleaved from the NW by incubating with $10 \mathrm{mM}$ dithiothreitol for 30 minutes at room temperature. The iron oxide was separated from the peptide by ultracentrifugation $\left(100,000 \times \mathrm{g}\right.$ for 10 minutes at $\left.4^{\circ} \mathrm{C}\right)$ and the 
supernatant fractions containing the peptide were analyzed by matrix-assisted laser desorption ionization-time of flight (MALDI-TOF) mass spectrometry.

Immunofluorescence and Immunohistochemistry. Tissues from mice injected with nanoparticles were fixed in $4 \%$ paraformaldehyde overnight at $4^{\circ} \mathrm{C}$, cryo-protected in $30 \%$ sucrose overnight and frozen in OCT embedding medium. For histological analysis, 7- $\mu \mathrm{m}$ sections were cut and sections were H\&E stained.

For immunostaining, tissue sections were first incubated for 1 hour at room temperature with $10 \%$ serum from the species in which the secondary antibody was generated, followed by incubation with the primary antibody overnight at $4^{\circ} \mathrm{C}$. The following antibodies were used: rat monoclonal anti-mouse CD31 (10 $\mathrm{g} / \mathrm{mL}$; BD Pharmingen, San Jose, CA), mouse fibrin(ogen) antiserum (1:100; Nordic, Tilburg, Netherlands). The primary antibodies were detected with Alexa 647 goat anti-rat, and 647 donkey anti-goat secondary antibodies (1:1000; Molecular Probes, Eugene, OR). Each staining experiment included sections stained with secondary antibodies only as negative controls. Nuclei were counterstained with DAPI $(5 \mu \mathrm{g} / \mathrm{mL}$; Molecular Probes). The sections were mounted in gel/mount mounting medium (Biomeda, Foster City, CA) and viewed under a Fluoview 500 confocal microscope (Olympus America, Center Valley, PA).

For immunohistochemical staining of frozen tissue sections, endogenous peroxidases were quenched with $3 \% \mathrm{H}_{2} \mathrm{O}_{2}$ (DAKO-Cytomation). Sections were blocked for 1 hour in $5 \%$ Donkey serum in Dako cytomation Ab dilution solution. Sections were treated with DAKO Biotin/Avidin Blocking system; incubated overnight at $4^{\circ} \mathrm{C}$ with mouse fibrin(ogen) antiserum. The primary antibody was detected with biotinylated anti-mouse lgG and Vectastain ABC kit (Vector Laboratories, Burlingame, CA). Nuclei were counterstained with hematoxylin (Vector Laboratories Burlingame, CA). To quantify the homing area of fibrin(ogen) within tumors the stained section were scanned with the Scanscope CM-1 scanner and analyzed with the ImageScope software41 (Aperio Technologies, Vista, CA).

Apoptosis was determined using the terminal deoxynucleotidyl transferase-mediated deoxyuridine triphosphate nick end labeling (TUNEL) assay for the identification of doublestranded DNA breaks using the In situ Cell Death Detection Kit (Roche Applied Science, Indianapolis, IN), according to the manufacturer's instructions. 
Magnetic resonance imaging. Nude mice bearing 22Rv-1 orthotopic human prostate cancer xenografts were intravenously injected with superparamagnetic iron oxide NW coated with peptides, or left not coated at a dose of $5 \mathrm{mg}$ of iron per $\mathrm{kg}$ body weight. Each animal received $\mathrm{Ni}$-liposomes $(0.2 \mathrm{mmol}$ of $\mathrm{Ni})$ intravenously 1 hour prior to the $\mathrm{NW}$ to increase the blood half-life of the NW. Approximately 7-8 hours after the NW injection, the mice were anesthetized with isoflurane and subjected to T2-weighted MRI scans with a 3-Tesla MR imager (GE Healthcare Technologies, Milwaukee, WI). After imaging, tissues of interest were harvested and processed for immunofluorecence.

Contrast-enhanced ultrasound (CEUS). Definity ${ }^{\circledR}$ contrast agent (Lantheus Medical Imaging; 5 $\mu \mathrm{l}$ in $45 \mu \mathrm{l}$ of saline) were injected into the tail vein of mice using a 28 gauge insulin syringe. Philips iU22 with a L12-5 transducer was used for imaging with a low mechanical index (MI), contrast specific imaging mode (power modulation mode). The imaging parameters were set as follows and kept identical throughout the study: depth $2 \mathrm{~cm}$, focus zone $2 \mathrm{~cm}$, Ml 0.06, 5 frames per second. Two-minute cine loop was saved for time intensity curve analysis using Philips QLab. CEUS was performed prior to, 1, 3, and 6 hours after injection of mice with CREKA-NW alone or in combination with CRKDKC-NW. The tumor rim, center, and the surrounding tissue were separately examined to quantify the efficiency of tumor circulation. CEUS analysis of tumors treated for 2 weeks with the NW was carried out using Photoshop with a threshold method, in which the pixels with enhancement and those without were counted (the threshold was set arbitrarily, as there was no fixed number). The percent area with enhancement $=$ the pixel number of the enhanced area/the pixel number of the entire tumor $x 100$.

Statistical analysis. Data were analyzed by two-tailed Student's unpaired t-test or one-way analysis of variance (ANOVA) followed by suitable post-hoc test. P values of less than 0.05 were considered statistically significant. 


\section{Results}

Nanoworm combinations for enhancing the activity of CREKA nanoparticles

We used elongated iron oxide nanoparticles, "nanoworms" (NW), which are more effective in peptide-mediated cell binding than spherical particles ${ }^{25}$. Nanoworms (NW) coated with the CREKA peptide accumulated in the vessels of orthotopic 22Rv1 human prostate cancer xenograft tumors and caused clotting in them, as evidenced by the presence of fibrin(ogen)containing deposits in the vessel lumens (Figure 1a). In tumors of untreated mice (not shown) or mice treated with nanoparticles coated with peptides other than CREKA, mainly the blood vessel walls were positive for fibrin(ogen) (Figure 1b). Most of the increased fibrin(ogen) staining in the CREKA-NW-treated 22Rv1 tumors was in the tumor periphery, whereas little NW accumulation or clotting was seen in the center of the tumors.

We hypothesized that it might be possible to improve the tumor delivery of NW by combining CREKA with a tumor-homing peptide that recognizes a different target molecule other than CREKA, and therefore potentially binds to different vessels in the tumor. We tested two tumorhoming peptides for their ability to increase CREKA-NW homing: CRKDKC, which was originally identified as a wound-homing peptide ${ }^{27}$, and CGKRK ${ }^{28}$. Both peptides bind to the blood vessels in various kinds of tumors ${ }^{27-28}$. Both CRKDKC-NW and CGKRK-NW accumulated in more than $70 \%$ of the $22 R v 1$ tumor vessels as evidenced by colocalization with CD31 staining (Figure 1b, Supplementary Figure 1).

We next tested NW double-coated with CREKA and one of the other peptides. However, the double-coated NW showed only minimal tumor homing (Supplementary Figure 2). It may be that coating the nanoparticles with two different peptides on the same nanoparticle reduces the surface density of each peptide to an extent that adversely affects the homing. Surprisingly, we found that mixtures of CREKA-NW with CRKDKC-NW or CGKRK-NW, which were used as controls, were quite effective in delivering CREKA-NW to vessels in the tumor interior and causing clotting in them. The mixed single-peptide particles may aggregate in tumor vessels, making it possible for each peptide to carry both types of particles to the target. Among the particle mixtures, the CREKA-CRKDKC mixture was particularly effective in homing to the entire tumor (Figure 1c), and we subsequently focused on this combination. Quantification of 
fibrin(ogen) accumulation showed that combining CREKA-NW with CRKDKC-NW enhanced clotting by 4-5 fold compared to CREKA-NW alone (Figure 1d). Contrast-Enhanced Ultrasound (CEUS) Imaging showed a 90\% reduction in blood flow at 6 hours after the injection of the NW mixture (Figure 2).

\section{Enhancing the activity of CREKA by incorporation of non-coded amino acids}

We hypothesized that protecting CREKA against proteolytic degradation may further increase the efficacy of the tumor homing. Proteolysis protection can be achieved through the incorporation of non-proteinogenic amino acids, provided that the bioactive conformation of the peptide is retained ${ }^{24,29}$. Based on molecular modeling studies ${ }^{24}$, several $\mathrm{N}$ - and $\mathrm{C} \alpha$-methylated amino acids were selected to replace the key residues in CREKA. This chemical modification can be viewed as the methylation in certain positions of the peptide backbone (at either $\mathrm{N}$ or $\mathrm{C} \alpha$ ). These studies indicated that the $\mathrm{N}$-methylation and $\mathrm{C}_{\alpha}$-methylation of the Arg, Glu and Lys residues does not affect the conformational profile of the peptide (Supplementary Figure 3). The CREKA analogs incorporating the $N / C \alpha$-methylated amino acids were synthesized as fluorescein (FAM)-labeled peptides. Several of the CREKA analogs showed significantly higher accumulation in the tumors than unmodified CREKA (Figure 3a). An exception was $\mathrm{C}(\mathrm{MMe}) \mathrm{REKA}$, in which the arginine residue is $\mathrm{N}$-methylated. This peptide gave only weak tumor fluorescence. Confocal microscopy confirmed the organ-level analyses (shown for $\mathrm{CR}(\mathrm{MMe}) \mathrm{EKA}$ in 22Rv1 tumors in Figure 3b and for LAPC9 tumors in Figure 3c). The active CREKA analogs displayed a meshwork pattern within both the viable and the necrotic lesions of tumor stroma that was stronger and more extensive than that seen with CREKA. As expected, staining of tumor sections with antibodies against fibrin(ogen) and fibronectin showed that the CREKA analogs accumulated in areas rich in deposition of these proteins (Figure 3b, panels on the right). $\mathrm{CR}(\mathrm{MMe}) \mathrm{EKA}$ and $\mathrm{CRE}(\mathrm{C} \alpha \mathrm{Me}) \mathrm{KA}$ appeared to be equally active in this regard (Figure 4).

To investigate the stability of CREKA and $\mathrm{CR}(M \mathrm{Me}) \mathrm{EKA}$ in the tumor in vivo, these peptides were intravenously injected into mice bearing 22Rv1 tumors. The peptides gave equally strong fluorescence in the tumors 30 minutes after the injection (Figure 3d). However, after 3 hours the CREKA fluorescence had decreased by $70 \%$, whereas $\mathrm{CR}(\mathrm{MMe}) \mathrm{EKA}$ showed no significant 
decrease, greater stability. Based on these observations, we selected the backbone-methylated peptides $\mathrm{CR}(\mathrm{MMe}) \mathrm{EKA}$ and $\mathrm{CRE}(\mathrm{C} \alpha \mathrm{Me}) \mathrm{KA}$ for studies with nanoparticles.

Intratumoral distribution of iron oxide nanoworms coated with CREKA analogs

Intravenously injected $\mathrm{CRE}(\mathrm{C} \alpha \mathrm{Me}) \mathrm{KA}-\mathrm{NW}$ and $\mathrm{CR}(\mathrm{MMe}) \mathrm{EKA}-\mathrm{NW}$ showed greatly enhanced accumulation in the blood vessels of the tumor rim and interior (Figure 4 and Figure 5a). These areas were also positive for anti-fibrin(ogen) staining. This distribution of the NW coated with the $N / C \alpha$-methylated peptides within tumors was markedly different from CREKA-NW, in which the nanoparticles appear less abundant in the interior of tumors, (compare Figures 4 and $5 \mathrm{a}$ with Figure 1a). No fluorescence from the various NW formulations was observed in normal tissues of the tumor-bearing mice, with the exception of the liver and the spleen, which non-selectively take up all nanoparticles. The liver accumulation of these NW was similar (shown for $\mathrm{CR}(\mathrm{MMe}) \mathrm{EKA}-\mathrm{NW}$ in Supplementary Figure 4). Significantly more tumor accumulation of $\mathrm{CR}(\mathrm{MMe}) \mathrm{EKA}-\mathrm{NW}$ than CREKA-NW was also observed in a different prostate cancer xenograft model, LAPC9 (Supplementary Figure 5).

Magnetic resonance imaging of 22Rv1 tumor mice after intravenous injection of NW showed hypointense vascular signals throughout the tumor for the $\mathrm{CR}(\mathrm{MMe}) \mathrm{EKA}-\mathrm{NW}$, and similar, but weaker signals with CREKA-NW (Figure 5b). Non-targeted nanoworms gave no detectable signal in these tumors after most of the nanoparticles had been cleared from the blood ${ }^{30}$.

Therapeutic efficacy of tumor blood vessel blockage by peptide-coated NW

Having optimized the CREKA self-amplified targeting system, by combining CREKA-NW with CRKDKC-NW and by incorporation of $N / C \alpha$-methylated amino acids, we tested the tumor treatment potential of the enhanced system. The Ni-liposome pre-treatment we used in the short-term experiments to block liver uptake of NW was not suitable for the long-term treatment experiments; it would have doubled the number of injections needed, and we also observed some deaths among mice that received multiple injections of Ni-liposomes. We omitted the liposome pre-treatment and instead coated the NW with polyethylene glycol (PEG) and coupled the homing peptide to the nanoparticles through the PEG chains. 
We used the peptides bound to the NW through PEG to directly compare the in vivo stability of CREKA and of $\mathrm{CR}(\mathrm{NMe}) \mathrm{EKA}$. The peptides were coupled through a reversible disulfide linkage to the PEG coating, and the coated NW were intravenously injected into mice bearing $22 \mathrm{Rv} 1$ tumors. The NW were recovered from the blood, and the peptides were isolated and analyzed by mass spectrometry. CREKA and $\mathrm{CR}(\mathrm{NMe}) \mathrm{EKA}$ were equally abundant in the blood samples obtained after 15 minutes of circulation (Supplementary Figure 6). However, after 3 hours, CREKA was undetectable, indicating that all the peptide was degraded, whereas the amount of $\mathrm{CR}(\mathrm{MMe})$ EKA only declined by about $60 \%$. These results show that, as intended by the chemical modification, $\mathrm{CR}(\mathrm{MMe}) \mathrm{EKA}$ is more stable in vivo than CREKA. CR(NMe)EKA bound to plasma clots with somewhat higher affinity than CREKA ( $\mathrm{Kd}=2.5 \mu \mathrm{M}$ and $6.0 \mu \mathrm{M}$; Supplementary Figure 7), suggesting that affinity may also contribute to the superior homing properties of the methylated peptide.

There was some loss of homing activity and clotting in tumor vessels compared to the PEG-free nanoparticles. We hypothesized that multiple injections might make up for this reduced activity. Indeed, many of the blood vessels around the necrotic area in the tumors of the mice treated for 2 weeks with the NW combination were filled with peptide particles and deposits positive for a fibrin(ogen) immunostaining (Figure 6a, Supplementary Figure 8). Moreover, CEUS (ContrastEnhanced Ultrasound) analysis of tumors treated with the NW revealed a reduction in tumor blood flow; CR(NMe)EKA-PEG-NW alone gave a 30\% reduction, and combining $\mathrm{CR}(\mathrm{MMe}) \mathrm{EKA}-$ PEG-NW with CRKDKC-PEG-NW more than doubled the effect (Figure 6b, Supplementary Figure 9).

Histological analysis of tumors from mice treated with $\mathrm{CR}(M \mathrm{Me})$ EKA-PEG-NW in combination with CRKDKC-PEG-NW showed extensive necrosis as evidenced by a typical loss of nuclei in the center of the tumors (Figure 6c). No signs of apoptosis or necrosis were observed in similarly sized tumors in the control groups (CRKDKC-PEG-NW and PBS). TUNEL staining revealed extensive apoptosis in the surviving areas of the tumors treated with $\mathrm{CR}(\mathrm{NMe}) \mathrm{EKA}$ PEG-NW and CRKDKC-PEG-NW combination (Figure 6d). There was highly significant inhibition of tumor growth in the mice treated with the combination compared with mice that received PBS or CRKDKC-PEG-NW alone (Figure 7a). CR(MMe)EKA-PEG-NW alone showed a modest but significant inhibition of tumor growth. The $\mathrm{CR}(\mathrm{NMe}) \mathrm{EKA}-\mathrm{PEG}-\mathrm{NW}$ and CRKDKCPEG-NW combination treatment also inhibited tumor growth in LAPC9 tumor mice, producing a 
significant survival increase compared to mice treated with vehicle alone (Figure 7b). These treatments showed no obvious systemic toxicity as evidenced by body weight measurements and histological analysis of organs from the treated mice. No signs of blood clotting elsewhere in the body (e.g. sudden death or paralysis due to stroke, pulmonary embolism, deep vein thrombosis) or signs of disseminated intravascular coagulation (DIC) were observed.

\section{Discussion}

Our results establish a tumor treatment and imaging strategy that is based on synergistic and self-amplifying accumulation of homing peptide-coated iron oxide NW in prostate tumor blood vessels. We show that extensive coverage of tumor vessels can be achieved by combining nanoparticles that were coated with either of two tumor-homing peptides, CREKA or CRKDKC. We also show that protecting the CREKA peptide against proteolysis through the incorporation of $N / C \alpha$-methylated residues increases the ability of the CREKA-NW to home to tumor blood vessels and to cause clotting in them. Combining the two approaches allowed effective MRI imaging of the tumors and produced extensive inhibition of tumor growth.

The changes we introduced into the CREKA self-amplifying tumor vessel homing system ${ }^{22}$ revealed surprising cooperation of two nanoparticle species coated with different peptides. We had assumed that to achieve greater coverage of prostate cancer vessels, the two peptides should be on the same particle, but found that having them on separate particles was more effective. Co-accumulation with CREKA-NW was not limited to the CRKDKC peptide; NW coated with another tumor-homing peptide, CGKRK, also enhanced the accumulation of CREKA-NW in tumor vessels, albeit not as strongly as CRKDKC-NW. A likely explanation for this phenomenon is co-aggregation of the two species of nanoparticles, which apparently only happens at the high concentrations of NW achieved within tumor vessels. The clotting induced by the CREKA-NW may contribute to the co-accumulation by trapping loosely bound CRKDKCNW that would otherwise be washed away by the blood flow. Another cooperative two-particle nanosystem has been described recently ${ }^{31}$.

The pro-thrombotic activity of the CREKA-nanoworms is selective for tumors. This is partly attributable to the selective accumulation of CREKA-NW, and the NW mixture, in tumor vessels. Both the peptide and the iron oxide component of the nanoparticles are responsible for the 
thrombotic activity, as other types of nanoparticles coated with CREKA and the free CREKA peptide lack this activity ${ }^{26,32}$, and iron oxide NW coated with tumor-homing peptides other than CREKA (CRKDKC and CGKRK) also do not cause clotting. Iron oxide nanoparticles are known to be procoagulant, and the affinity of CREKA for clotting products apparently enhances that activity. The NW, like other nanoparticles, non-specifically accumulate in the liver and spleen ${ }^{33}$. However, we have not observed any clotting in the normal tissues of the tumor mice in this study, or in an earlier study that used a similar but less effective system ${ }^{22}$. Thus, the tumor environment, which is pro-coagulant ${ }^{19-20}$, is also likely to play a role in bringing about the clotting in the tumor vessels. Atherosclerotic plaques tend to show subtle spontaneous clotting 32,34 . Thus, the presence of atherosclerosis could be a limitation in clinical application of the CREKA technology.

The potential of peptides as drug candidates is limited by poor pharmacokinetics, which includes rapid elimination from the circulation through filtration into the urine and susceptibility to proteolysis. Elimination into the urine is not a problem with peptides coated onto a nanoparticle, but our results do demonstrate the importance of proteolysis. Because each nanoparticle is coated with multiple peptides, one might expect the nanoparticle coating to tolerate some proteolysis without significant loss of activity. Our results indicate that this is not the case; protecting the peptide against proteolytic cleavage by incorporating non-coded amino acids substantially improved the tumor-homing efficiency and increased the blockade of tumor vessels. Direct measurement of the stability of the peptides in vivo supported the conclusion that increased peptide stability is responsible, at least partially, for the improved characteristics of the methylated CREKA analogs. If extended to other peptide-coated nanoparticles, this result could have significant implications for the design of effective nanoparticle therapies.

Here we show anti-tumor activity of our system against two orthotropic prostate cancer xenograft tumors, 22Rv-1 and LAPC9. It is likely that other tumor types can be similarly targeted, as we have shown other types of tumors to be targeted by the CREKA system ${ }^{22}$. The efficiency of the combined CREKA-NW strongly correlated with the degree of tumor vessel blockade achieved with the various treatments. CR(MMe)EKA-PEG-NW caused $30 \%$ reduction in tumor blood flow, as documented by CEUS, and produced a modest reduction in tumor size. The CRKDKC-PEG-NW and CR(NMe)EKA-PEG-NW combination blocked $70 \%$ of tumor blood flow and gave a strong reduction in tumor size, as well as extended survival of the animals. 
Significantly, these results were obtained by utilizing only the inherent properties of the NW, which also allowed imaging of the tumors. Adding a drug to the particles could further enhance the utility of this theranostic nanosystem. 


\section{Acknowledgements}

We thank Dr. Jerry Lee for discussions. Jacqueline Corbeil helped with MRI, Dr. Gema Ballano with the syntheses and Guillem Revilla-Lopez with simulations. Centre de Supercomputacio de Catalunya and the Barcelona Supercomputing Center provided computational facilities. This work was supported by NCI grants P01 (CA104898 (E.R.), CA124427 (M.J.S., E.R.) and CA119335 (R.F.M., M.J.S., E.R.), and Cancer Center Support Grant CA 30199, P50-CA128346 to UCSD ICMIC Center, and grants CTQ2008-00423-E/BQU and CTQ2007-62245 (A.I.J., C.C., C.A.) from the Spanish Ministerio de Ciencia e Innovacion. This project has been funded in part with Federal funds from the National Cancer Institute, $\mathrm{NIH}$, under contract number HHSN261200800001E. The content of this publication does not necessarily reflect the views or policies of the Department of Health and Human Services, nor does mention of trade names, commercial products or organizations imply endorsement by the US Government. This research was supported (in part) by the Intramural Research Program of the NIH, National Cancer Institute, Center for Cancer Research.

\section{Author contribution}

L.A., K.N.S., A.I.J, C.C., C.A., R.N. and E.R. designed the experiments, L.A. and K.N.S. conducted the biochemical and animal experiments, L.A., K.G. and V.R.K. synthesized nanoworms. V.R.K. participated in the design of the synthetic peptides, performed the syntheses, and analyzed the mass spectrometry data. K.N.S, O.G., Y.K. and R.F.M provided the imaging technologies. M.J.S and J.H.P designed the iron oxide nanoworms F.J.S., A.I.J, C.C., D.S., C.A. and R.N. performed computer simulation analyses on the structure of CREKA, helped design the $N / C$ methylated peptides and provided the $N / C$ methylated amino acid precursors for peptide synthesis. F.J.S., A.I.J, C.C., D.Z., C.A. and R.N. performed computer simulation analyses on the structure of CREKA, designed the peptide analogs and provided the N/Ca-methylated amino acids. L.A. and E.R. wrote the manuscript. All authors discussed the results and commented on the manuscript.

Financial Interests statement

The authors state that there is no financial conflict of interest in the publication of this paper. 


\section{References}

1. Jemal A, Siegel R, Ward E, et al. Cancer statistics, 2008. CA Cancer J Clin. 2008;58(2):71-96.

2. Macintosh CA, Stower M, Reid N, Maitland NJ. Precise microdissection of human prostate cancers reveals genotypic heterogeneity. Cancer Res. 1998;58(1):23-28.

3. Ruijter ET, van de Kaa CA, Schalken JA, Debruyne FM, Ruiter DJ. Histological grade heterogeneity in multifocal prostate cancer. Biological and clinical implications. J Pathol. 1996;180(3):295-299.

4. Miller GJ, Cygan JM. Morphology of prostate cancer: the effects of multifocality on histological grade, tumor volume and capsule penetration. J Urol. 1994;152(5 Pt 2):1709-1713.

5. Jain RK, Munn LL, Fukumura D. Dissecting tumour pathophysiology using intravital microscopy. Nat Rev Cancer. 2002;2(4):266-276.

6. Weissleder R. Scaling down imaging: molecular mapping of cancer in mice. Nat Rev Cancer. 2002;2(1):11-18.

7. Ruoslahti E, Bhatia, S.N. and Sailor, M.J. . Targeting of Drugs and Nanoparticles to Tumors. J. Cell Biol. In press. 2010.

8. Folkman J. Angiogenesis in cancer, vascular, rheumatoid and other disease. Nat Med. 1995;1(1):27-31.

9. Ferrara N, Alitalo K. Clinical applications of angiogenic growth factors and their inhibitors. Nat Med. 1999;5(12):1359-1364.

10. Folkman J. Angiogenesis: an organizing principle for drug discovery? Nat Rev Drug Discov. 2007;6(4):273-286.

11. Carmeliet P. Mechanisms of angiogenesis and arteriogenesis. Nat Med. 2000;6(4):389-395.

12. Beecken WD, Fernandez A, Joussen AM, et al. Effect of antiangiogenic therapy on slowly growing, poorly vascularized tumors in mice. J Natl Cancer Inst. 2001;93(5):382-387.

13. Bieker R, Kessler T, Schwoppe $C$, et al. Infarction of tumor vessels by NGR-peptide-directed targeting of tissue factor: experimental results and first-in-man experience. Blood. 2009;113(20):5019-5027.

14. Huang X, Molema G, King S, Watkins L, Edgington TS, Thorpe PE. Tumor infarction in mice by antibody-directed targeting of tissue factor to tumor vasculature. Science. 1997;275(5299):547550.

15. Kessler T, Bieker R, Padro T, et al. Inhibition of tumor growth by RGD peptide-directed delivery of truncated tissue factor to the tumor vasculature. Clin Cancer Res. 2005;11(17):6317-6324.

16. Nilsson F, Kosmehl H, Zardi L, Neri D. Targeted delivery of tissue factor to the ED-B domain of fibronectin, a marker of angiogenesis, mediates the infarction of solid tumors in mice. Cancer Res. 2001;61(2):711-716.

17. Ran S, Gao B, Duffy S, Watkins L, Rote N, Thorpe PE. Infarction of solid Hodgkin's tumors in mice by antibody-directed targeting of tissue factor to tumor vasculature. Cancer Res. 1998;58(20):4646-4653.

18. Hoffman JA, Laakkonen, P., Porkka, K., Bernasconi, M., and Ruoslahti, E. In vivo and ex vivo selections using phage-displayed libraries. In Phage Display: A Practical Approach. Oxford University Press. . 2004; Chap 10:171.

19. Abe K, Shoji M, Chen J, et al. Regulation of vascular endothelial growth factor production and angiogenesis by the cytoplasmic tail of tissue factor. Proc Natl Acad Sci U S A. 1999;96(15):86638668.

20. Dvorak HF, Senger DR, Dvorak AM, Harvey VS, McDonagh J. Regulation of extravascular coagulation by microvascular permeability. Science. 1985;227(4690):1059-1061. 
21. Pilch J, Brown DM, Komatsu M, et al. Peptides selected for binding to clotted plasma accumulate in tumor stroma and wounds. Proc Natl Acad Sci U S A. 2006;103(8):2800-2804.

22. Simberg D, Duza T, Park JH, et al. Biomimetic amplification of nanoparticle homing to tumors. Proc Natl Acad Sci U S A. 2007;104(3):932-936.

23. Agemy L, Harmelin A, Waks T, et al. Irradiation enhances the metastatic potential of prostatic small cell carcinoma xenografts. Prostate. 2008;68(5):530-539.

24. Zanuy $\mathrm{D}$, Flores-Ortega A, Jimenez Al, et al. In silico molecular engineering for a targeted replacement in a tumor-homing peptide. J Phys Chem B. 2009;113(22):7879-7889.

25. Park JH, von Maltzahn $\mathrm{G}$, Zhang L, et al. Systematic surface engineering of magnetic nanoworms for in vivo tumor targeting. Small. 2009;5(6):694-700.

26. Jarvinen TA, Ruoslahti E. Molecular changes in the vasculature of injured tissues. Am J Pathol. 2007;171(2):702-711.

27. Hoffman JA, Giraudo E, Singh M, et al. Progressive vascular changes in a transgenic mouse model of squamous cell carcinoma. Cancer Cell. 2003;4(5):383-391.

28. Chatterjee J, Gilon C, Hoffman A, Kessler H. N-methylation of peptides: a new perspective in medicinal chemistry. Acc Chem Res. 2008;41(10):1331-1342.

29. Sugahara KN, Teesalu T, Karmali PP, et al. Tissue-penetrating delivery of compounds and nanoparticles into tumors. Cancer Cell. 2009;16(6):510-520.

30. Park JH, von Maltzahn G, Xu MJ, et al. Cooperative nanomaterial system to sensitize, target, and treat tumors. Proc Natl Acad Sci U S A. Dec 282009.

31. Karmali PP, Kotamraju VR, Kastantin M, et al. Targeting of albumin-embedded paclitaxel nanoparticles to tumors. Nanomedicine. 2009;5(1):73-82.

32. Peters $D$, Kastantin $M$, Kotamraju VR, et al. Targeting atherosclerosis by using modular, multifunctional micelles. Proc Natl Acad Sci U S A. 2009;106(24):9815-9819.

33. Thorek DL, Chen AK, Czupryna J, Tsourkas A. Superparamagnetic iron oxide nanoparticle probes for molecular imaging. Annals of biomedical engineering. 2006;34(1):23-38.

34. Smith EB. Fibrinogen and atherosclerosis. Wien Klin Wochenschr. 1993;105(15):417-424.

\section{Figure legends}

Figure 1. Combining CREKA NW with NW coated with another tumor-homing peptide enhances homing efficiency. a, Iron oxide NW coated with FAM-labeled CREKA peptide were intravenously injected (5 mg iron per kg body weight) into nude mice bearing orthotropic 22Rv1, human prostate tumors. The mice had been pre-injected with $\mathrm{Ni}$-liposomes to reduce uptake by the reticulo-endothelial system ${ }^{22}$. Tumors were harvested 5 hours later, and tumor sections were stained with antibodies and examined by confocal microscopy (the 5 hours time point was found to be optimal for NW homing with regard to accumulation of the NW in the tumor and clearance of the NW from the blood). The CREKA-coated particles are green; blood vessels and clotting were separately visualized with anti-CD31 (magenta) or anti-fibrin(ogen) staining (red); nuclei were stained with DAPI (blue). Scale bars, $200 \mu \mathrm{m}$. b, NW coated with FAM-labeled 
CRKDKC or CGKRK were intravenously injected and the tissues were collected and processed as in (a). CRKDKC or CGKRK-coated particles are green, blood vessels visualized with antiCD31 (magenta; the white color indicates colocalization of magenta and green) or antifibrin(ogen) staining (red; the yellow color indicates colocalization of red and green); nuclei were stained with DAPI (blue). Large vessels were selected for the right panels because intravascular clotting (which is not promoted by CRKDKC-NW or CGKRK -NW) is most apparent in larger vessels. Scale bars, $200 \mu \mathrm{m}$ in the left and middle panels; $100 \mu \mathrm{m}$ in the right panels. C, A mixture of NW coated with rhodamine-labeled CREKA (red) and FAM-labeled CRKDKC (green) was intravenously injected (2.5 mg iron/kg of each NW preparation), and the tissues were collected and processed as in (a) and stained for fibrin(ogen) (magenta); nuclei were stained with DAPI (blue). Scale bars, $200 \mu \mathrm{m}$. d, Mice were injected with the indicated materials as in (a), (b) or (c). The sections stained with anti-fibrin(ogen) antibody were subjected to image analysis using Scanscope to quantify fibrin(ogen)-positive areas. The insets show examples of anti-fibrin(ogen) immunostaining in the tumor rim (left) and interior (right) from mice injected with the NW mixture. Scale bars, $50 \mu \mathrm{m}$. Statistical analyses were performed with ANOVA. Error bars, mean \pm SEM $(n=5-6)$; double asterisk, $p<0.01$.

Figure 2. Contrast Enhanced Ultrasound (CEUS) imaging of blood circulation in tumors of mice treated with peptide-coated NW. a, Mice pre-injected with Ni-liposomes were subsequently injected with a mixture of CREKA-NW and CRKDKC-NW and, after the indicated periods of time, injected with an ultrasound contrast agent. Contrast-enhanced and conventional ultrasound (US) images obtained at the different time points are shown. The images are representative of 3 tumors imaged. b, Enhancement analysis curves of blood flow in different tumor regions and the surrounding tissue from experiments described in (a). The orientation of the tumors is slightly different between the time points because the mice were anesthetized for each scan and reintroduced to the US instrument. $n=3$

Figure 3. Tumor accumulation of the CREKA peptide and its $N / C \alpha$-methylated variants. Mice bearing orthotropic 22Rv1 xenograft tumors were intravenously injected with $200 \mu \mathrm{g}$ of FAMlabeled CREKA or N/C $\alpha$-methylated CREKA peptides and allowed to circulate for 3 hours. This time point highlights the differences between non-modified CREKA and some of the methylated variants (see panel $d$ ). The mice were perfused through the heart with PBS, and the organs shown were collected and viewed under UV light. a, Quantification of fluorescence with Image $J$ 
software. Several N/C $\alpha$-methylated CREKA analogs produced stronger fluorescence than unmodified CREKA. Statistical analyses were performed with ANOVA. Error bars show mean \pm SEM ( $n=3-4)$; double asterisk, $p<0.01$; triple asterisk $p<0.001$. Representative images from mice injected with the CREKA or CR(NMe)EKA peptides b, 22Rv-1 and c, LAPC9 xenografts. In the upper panels, white dotted lines show where the organs were placed in a macroscopic examination, and the yellow line outlines the tumor. The lower panels show confocal images of tumor sections from mice injected with the peptides (green) indicated above. Blood vessels were visualized with anti-CD31 (red); nuclei were stained with DAPI (blue). Scale bars, $200 \mu \mathrm{m}$. The right panels (b) show representative confocal image fields to illustrate the localization of the $\mathrm{CR}(\mathrm{MMe}) \mathrm{EKA}$ peptide (green) in relation to anti-fibrin(ogen) (red) and anti-fibronectin (magenta) staining used as markers of tumor stroma; nuclei were stained with DAPI (blue). Scale bar, 50 $\mu \mathrm{m}$. The right panels (c) show quantification of fluorescence with Image $\mathrm{J}$ software. Statistical analysis was performed with Student's t-test. Error bars show mean \pm SEM $(n=3)$; double asterisk, $p<0.01$. d, Quantification of fluorescence with Image $\mathrm{J}$ software 15 minutes or 3 hours after peptide injection to 22Rv1 tumor mice. CR(NMe)EKA produced stronger fluorescence over time compared to unmodified CREKA. Statistical analysis was performed with Student's t-test. Error bars show mean \pm SEM $(n=3-4)$; triple asterisk $\mathrm{p}<0.001$. (NMe) and $(C M e)$ indicate an $N$ or C $\alpha$-methylated residue, respectively.

Figure 4. $C R E(C M e) K A-N W$ homing to $22 R v 1$ tumors. Mice bearing orthotropic $22 \mathrm{Rv} 1$ xenograft tumors were intravenously injected with $200 \mu \mathrm{g}$ of FAM-labeled CRE(CMe)KA or $5 \mathrm{mg}$ iron/kg of NW coated with FAM-CRE(CMe)KA. The peptide was allowed to circulate for 3 hours and NW were allowed to circulate for 5 hours. The mice were then perfused through the heart with PBS, and the tumors were collected. Tumor sections were stained with CD31 or antifibrin(ogen) (red) and examined by confocal microscopy. NW are green; nuclei were stained with DAPI (blue). Scale bars, $200 \mu \mathrm{m} . n=3$.

Figure 5. Improved tumor homing of NW coated with an N-methylated CREKA peptide analog. NW coated with FAM-labeled CREKA peptide or its $N$-methylated variant, $\mathrm{CR}(\mathrm{MMe}) \mathrm{EKA}$, were intravenously injected into mice bearing 22Rv1 tumors (total dose $5 \mathrm{mg}$ iron/kg). a, Tumors were harvested 5 hours later, and tumor sections were stained with antibodies and examined by confocal microscopy. CR(MMe)EKA-NW are green; blood vessels and clotting were separately visualized with anti-CD31 or anti-fibrin(ogen) staining (red). Nuclei were stained with DAPI 
(blue). Scale bars, $100 \mu \mathrm{m}$ (50 $\mu \mathrm{m}$ in the inset). b, T2-weighted MR images (Fast Spin Echo, $\mathrm{TR}=6.4 \mathrm{~s}, \mathrm{TE}=69 \mathrm{~ms})$. CREKA-NW or $\mathrm{CR}(\mathrm{NMe}) \mathrm{EKA}-\mathrm{NW}$ were intravenously injected into tumorbearing mice. The particles were allowed to circulate for 7-8 hours (the time determined in preliminary experiments to be optimal for differential homing). Gray scale images of axial planes through the tumors are shown. Gadolinium $(\mathrm{Gd})$ and Feridex (Fe) were used as reference standards. $n=3-4$.

Figure 6. NW distribution and effects on intravascular clotting, tumor apoptosis and tumor therapy. Mice bearing 2 week-old orthotopic xenografts of 22Rv1 human prostate cancer were intravenously injected with NW coated with peptides through a 5-kDa polyethylene glycol spacer. The NW were administered every other day for 14 days (5 mg iron/kg/day, total cumulative dose $35 \mathrm{mg} / \mathrm{kg}$ ). a, Tumor sections were stained with anti-CD31 (red); CR(NMe)EKA-NW/CRKDKC-NW combination (green); nuclei were stained with DAPI (blue). Scale bars, $200 \mu \mathrm{m}$. Note: The necrotic area at the center of the tumor is autofluorescent. b, CEUS imaging and analysis showing reduction in tumor blood flow at the end of the treatment. The image are representative of $n=3$. c, H\&E staining showing a large necrotic area (arrow) in the middle of a typical tumor treated with the CR(NMe)EKA-NW/CRKDKC-NW combination and occluded vessels in the viable rim of these tumors (broken arrows). A tumor of a similar size from a mouse treated with CRKDKC-NW alone is shown for comparison. d. Apoptosis analysis by TUNEL staining (red); NW combination (green); nuclei were stained with DAPI. Scale bars, $200 \mu \mathrm{m}$.

Figure 7. Tumor treatment with targeted nanoworms. Mice bearing orthotopic xenografts of 22Rv1 or LAPC9 human prostate cancer (2 weeks or 10 days after the inoculation, respectively) were intravenously injected with NW coated with peptides through a 5-kDa polyethylene glycol spacer. The particles were administered every other day for 14 days ( $5 \mathrm{mg}$ iron/ $\mathrm{kg} / \mathrm{day}$, total cumulative dose $35 \mathrm{mg} / \mathrm{kg}$ ). a, Tumor volume one day after the last injection in the 22Rv1 model is shown. Statistical analyses were performed with ANOVA. Error bars show mean \pm SEM ( $n=10-12)$; double asterisk, $p<0.01$; triple asterisk $p<0.001$. Similar results were obtained in two independent experiments. b, Mice bearing LAPC9 tumors were treated as described in (a) and survival was monitored over time ( $n=8$ per group). The arrow indicates the day the NW treatment was stopped. 
2010-274258 Agemy et al. Fig. 1
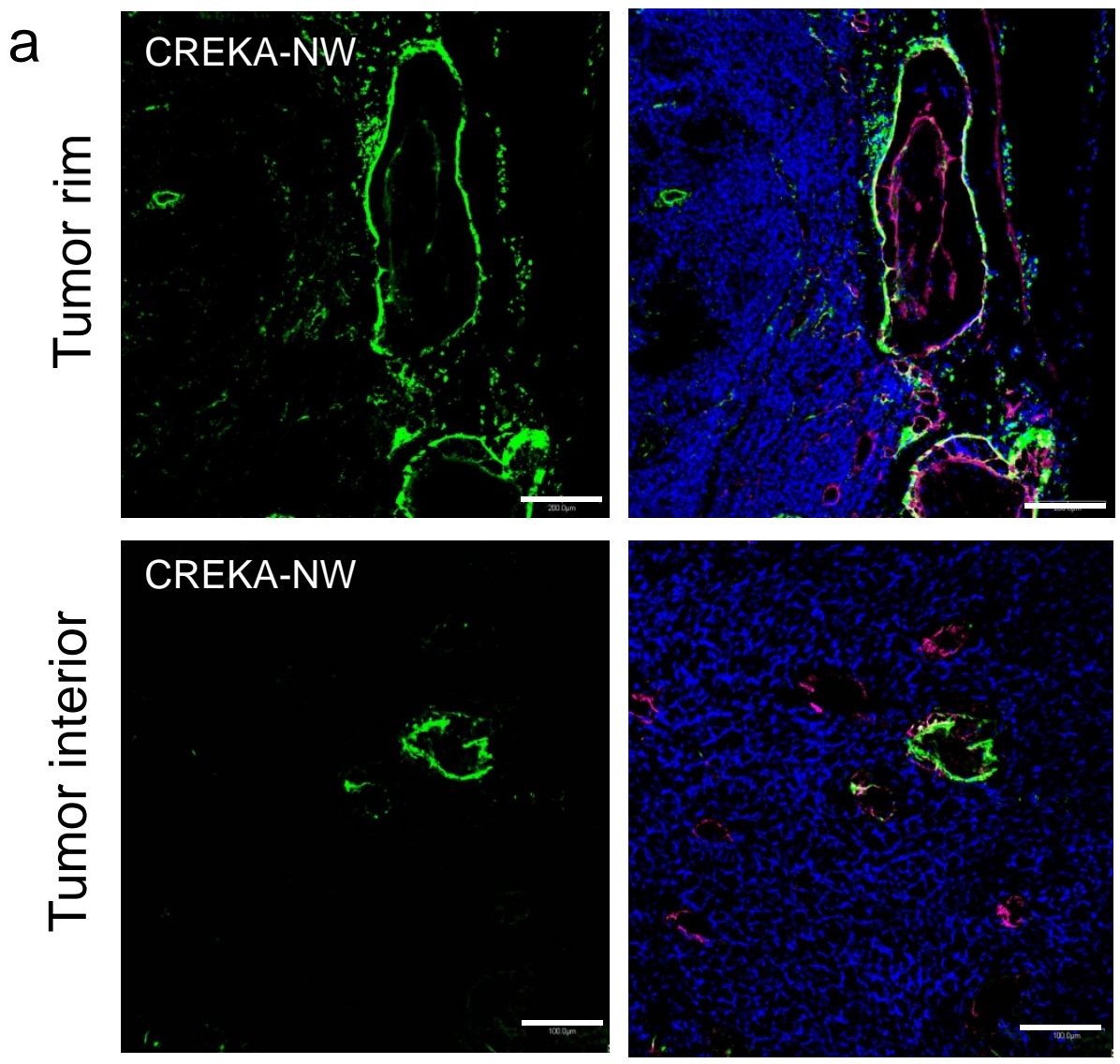

b

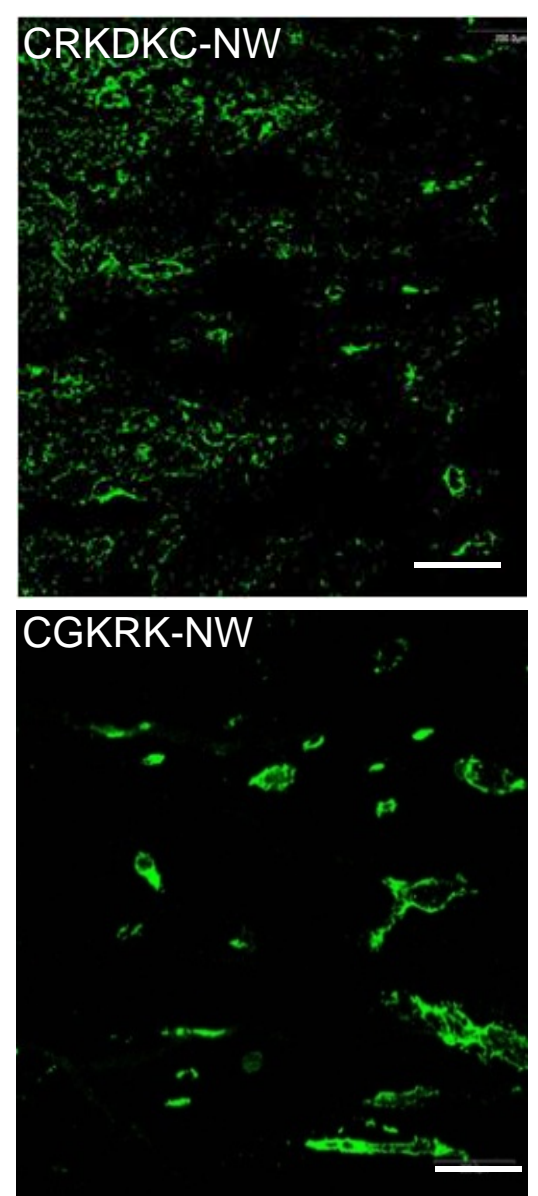

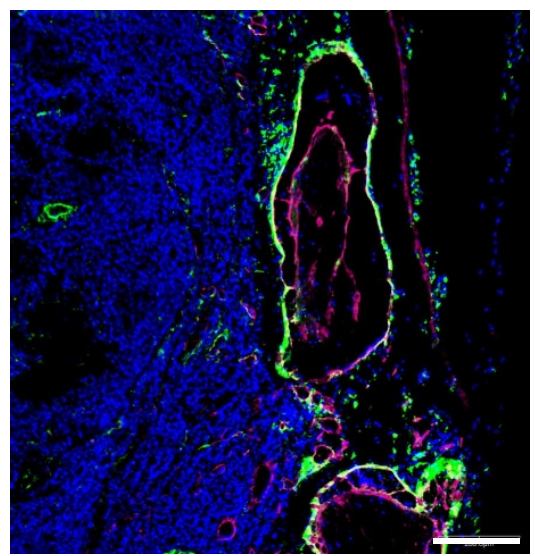

CD31

Fibrin(ogen)
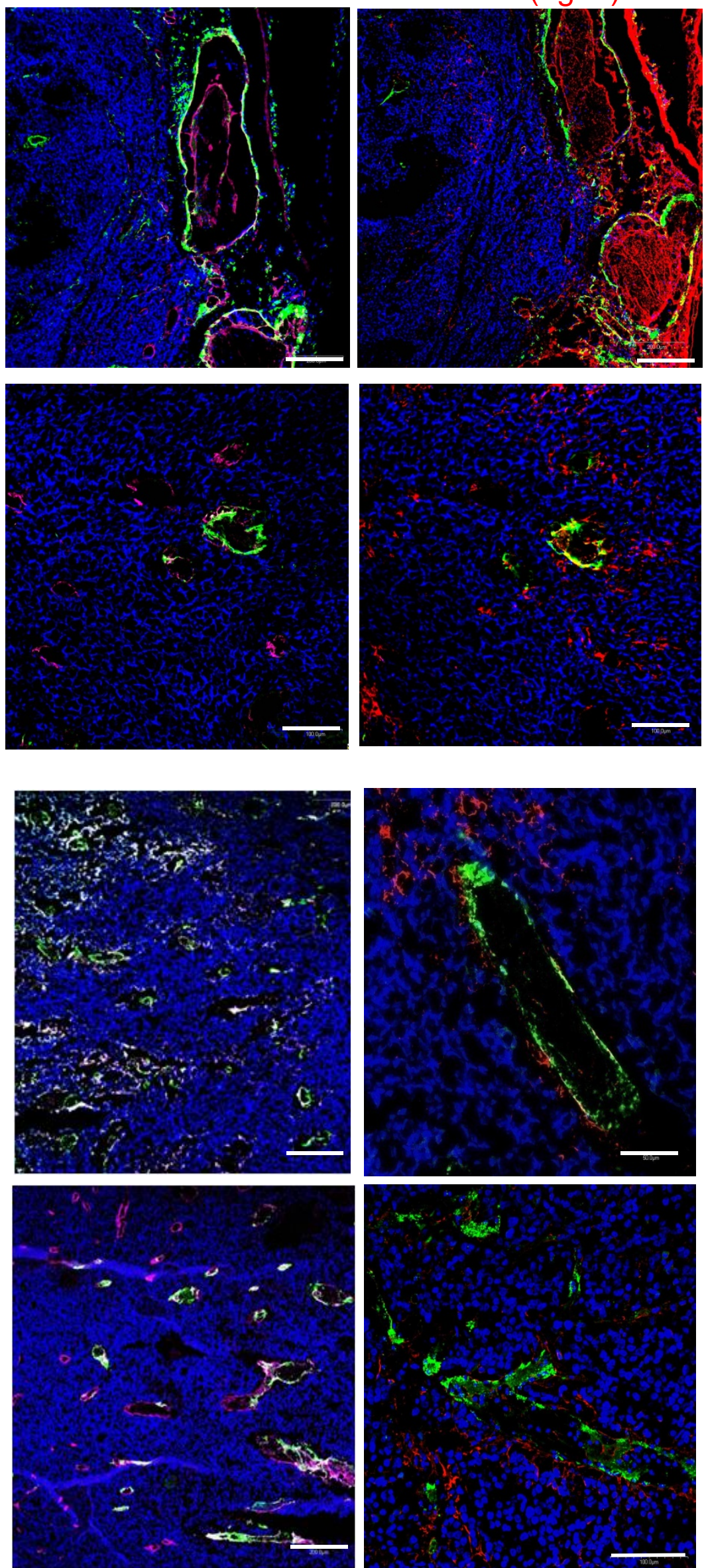
C

CREKA-NW
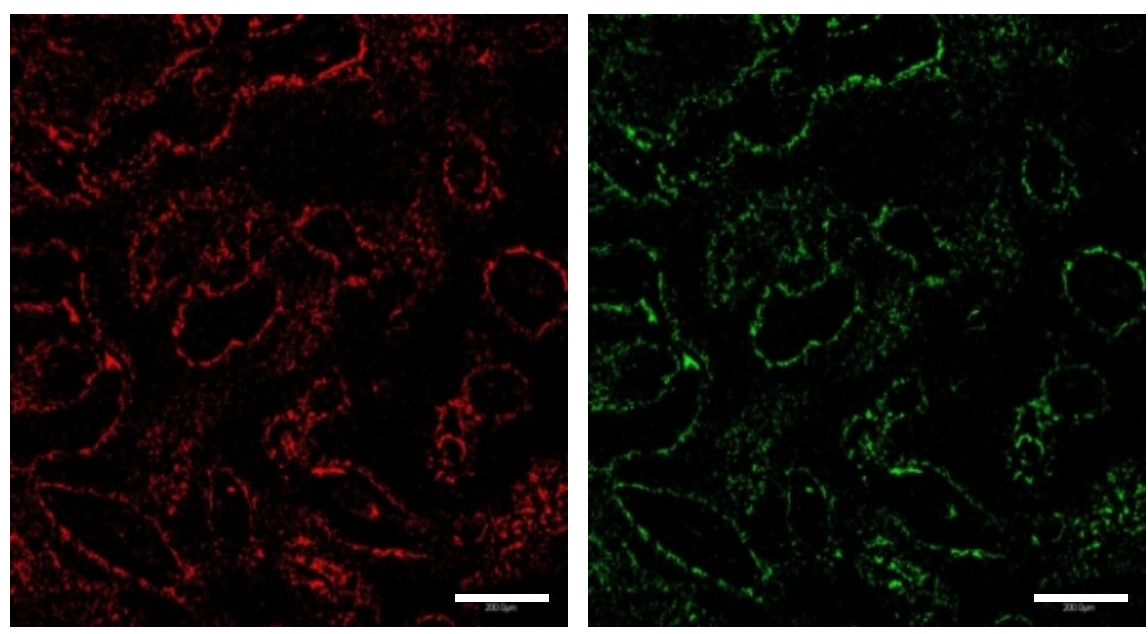

CRKDKC-NW

+fibrin(ogen)+DAPI

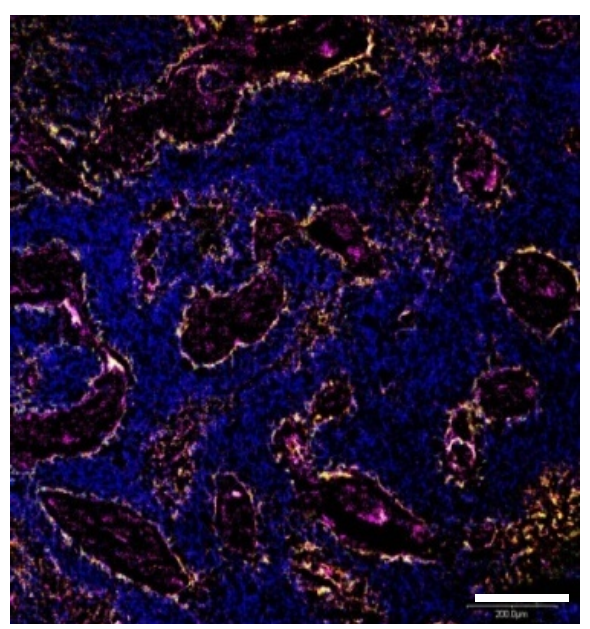

Tumor rim

d

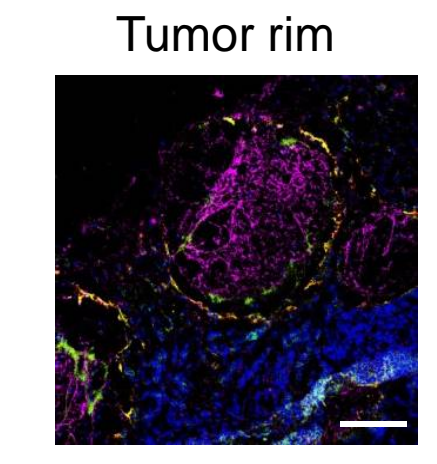

Tumor interior
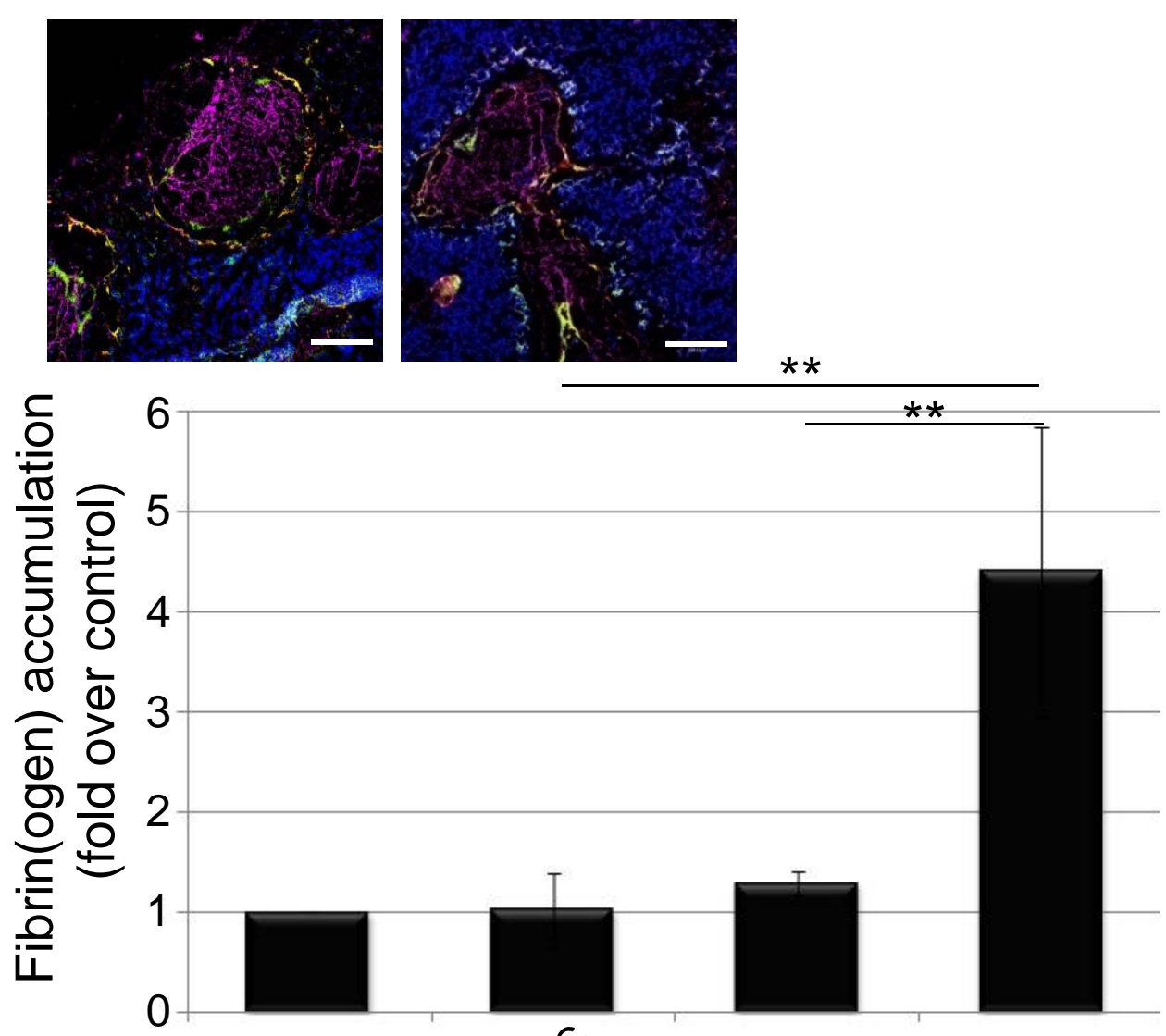

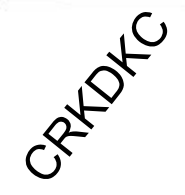

NW-peptide

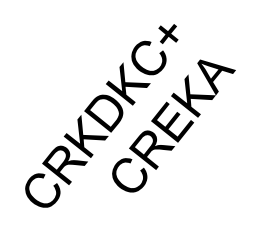


a

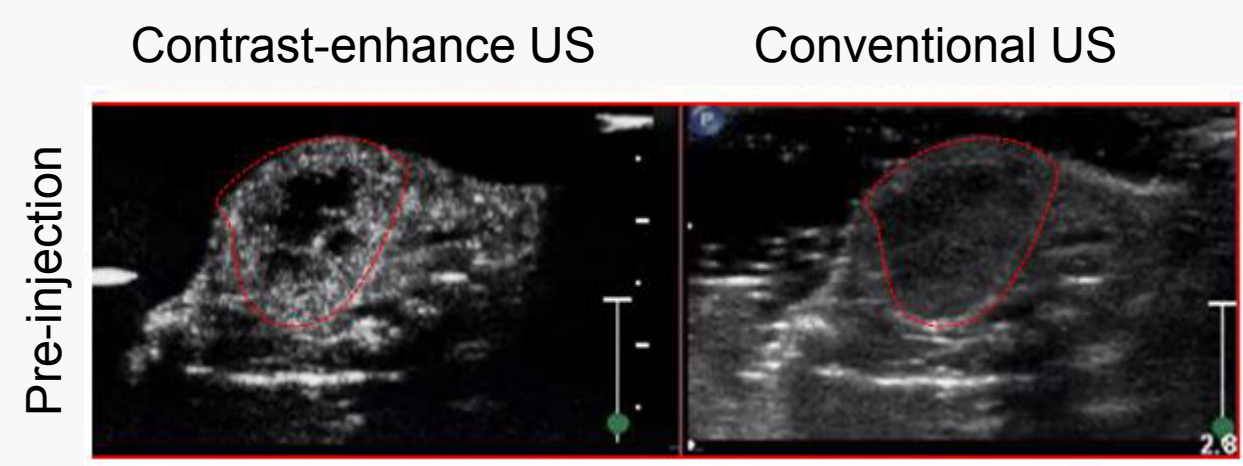

$1 \mathrm{~h}$

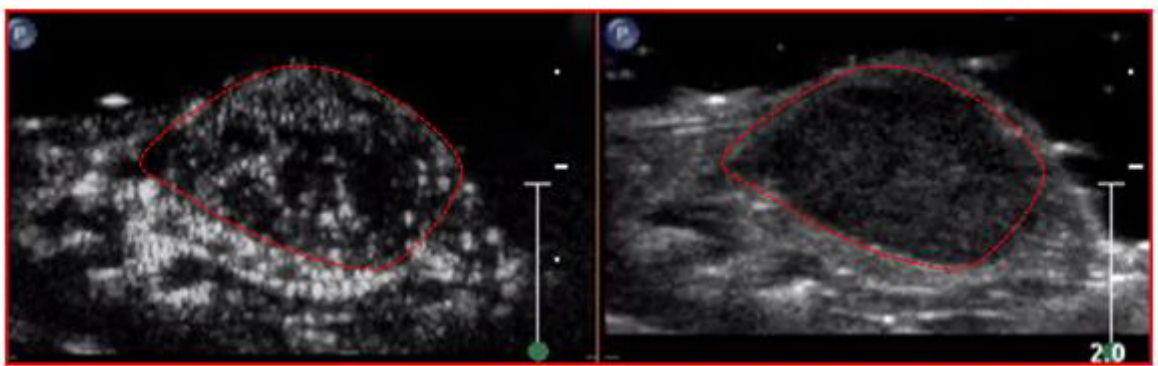

$3 \mathrm{~h}$

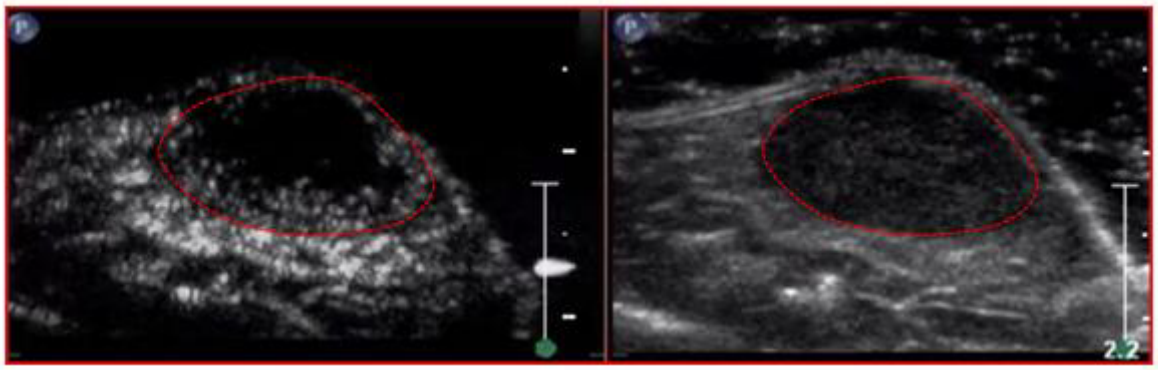

$6 \mathrm{~h}$

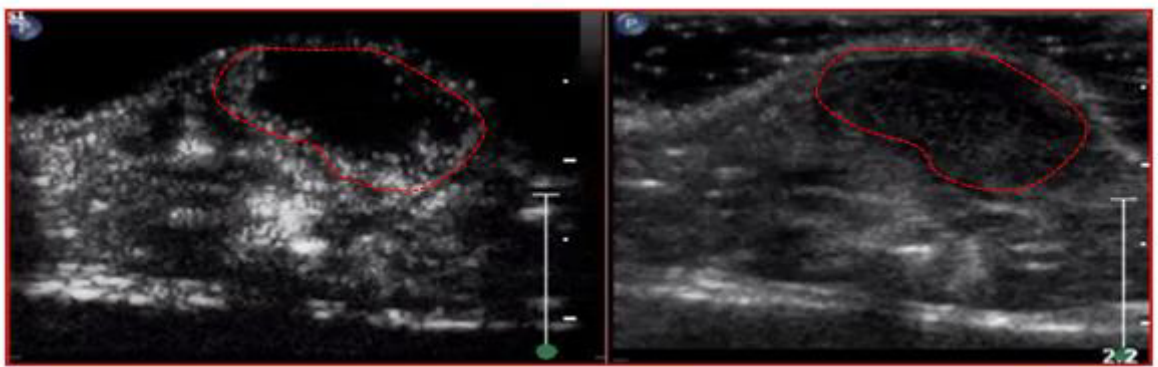

b.

Pre-injection

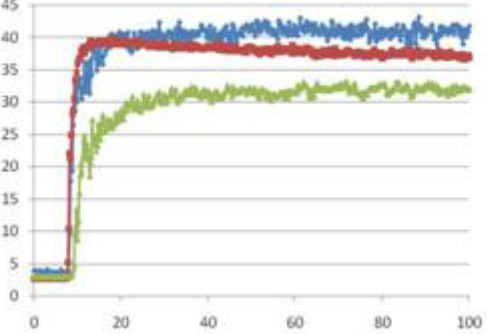

$\rightarrow-$ Surrounding tissue

- -Tumor rim

-Tumor interior

$1 \mathrm{~h}$

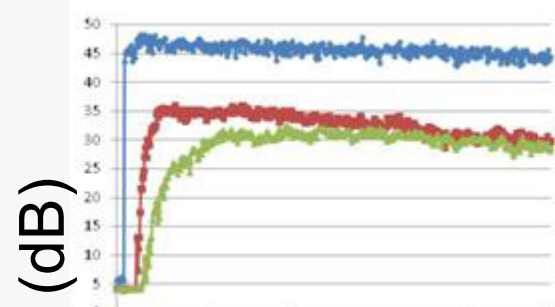

$3 \mathrm{~h}$
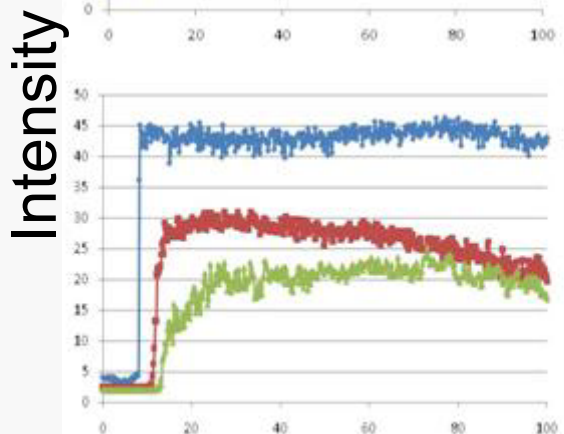

$6 \mathrm{~h}$

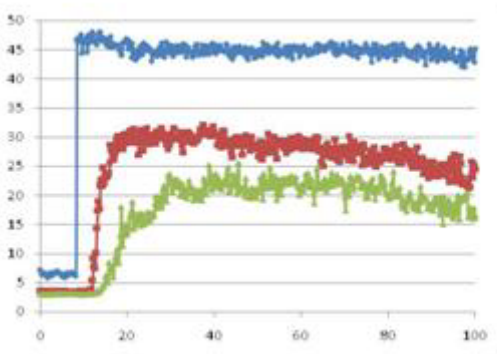

Time (seconds) 
CREKA
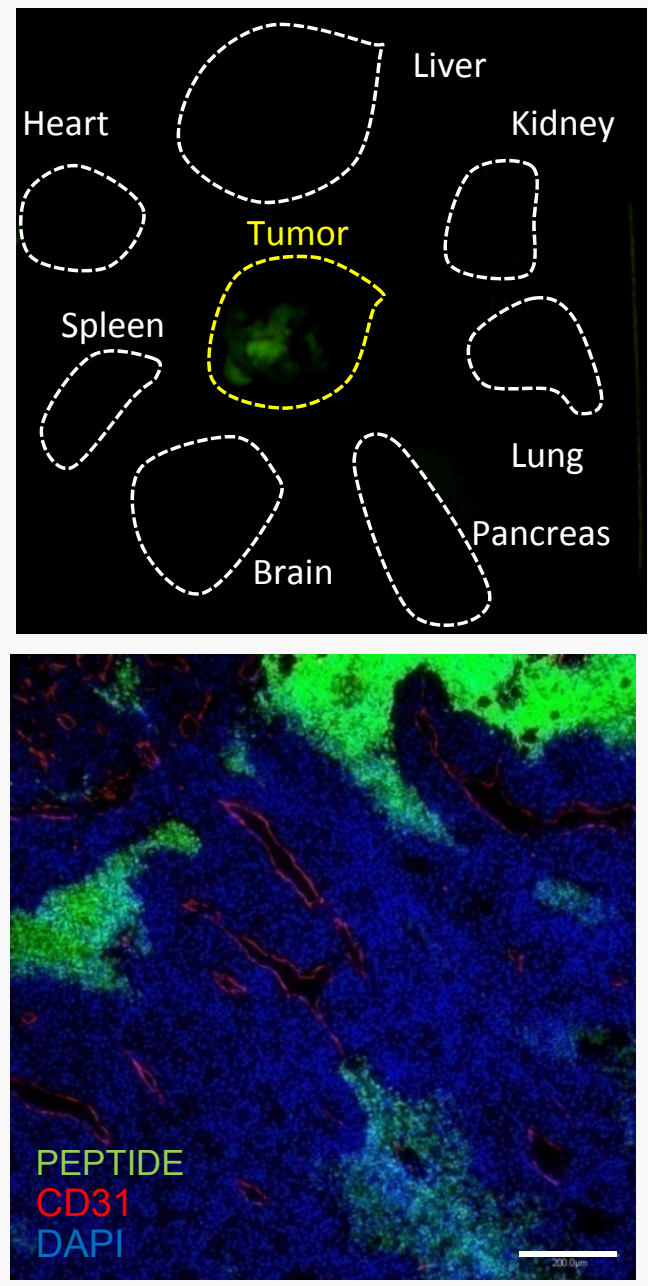

CR(NMe)EKA
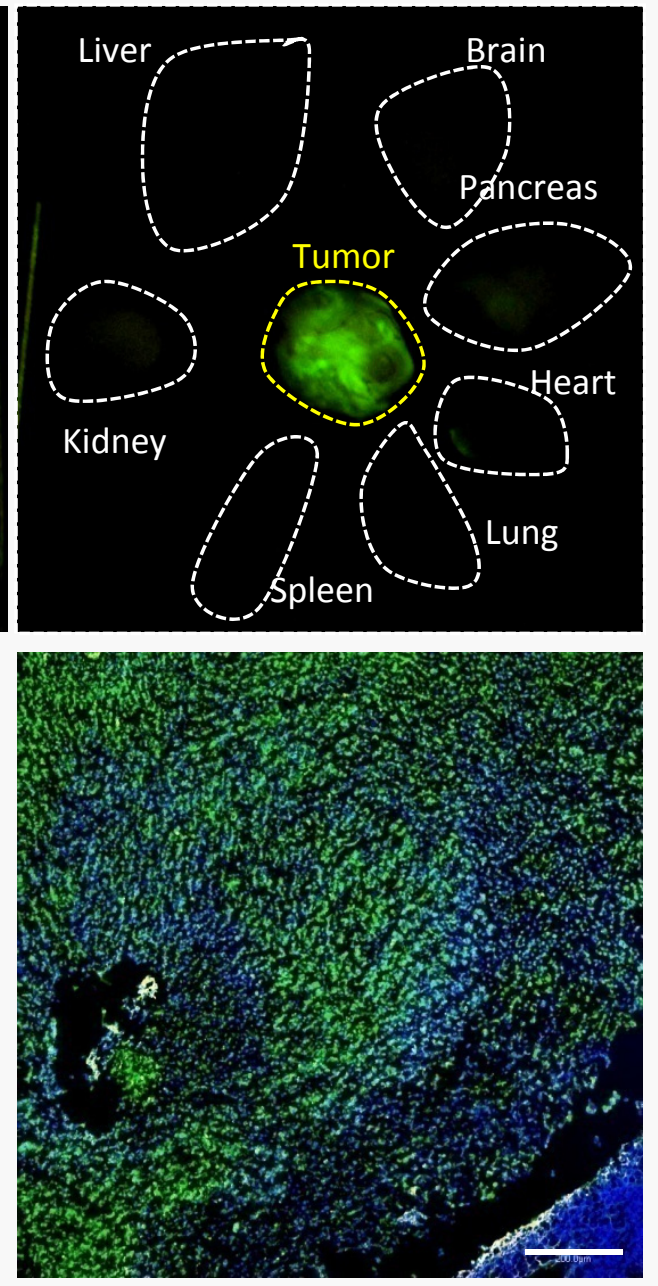

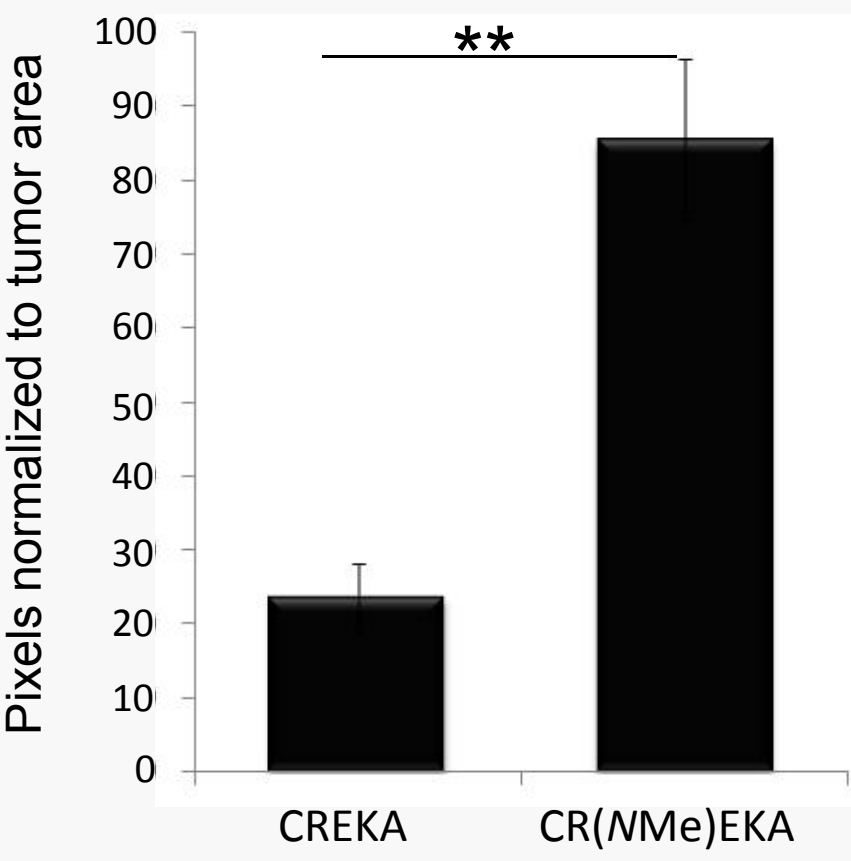


2010-274258 Agemy et al. Fig. 3

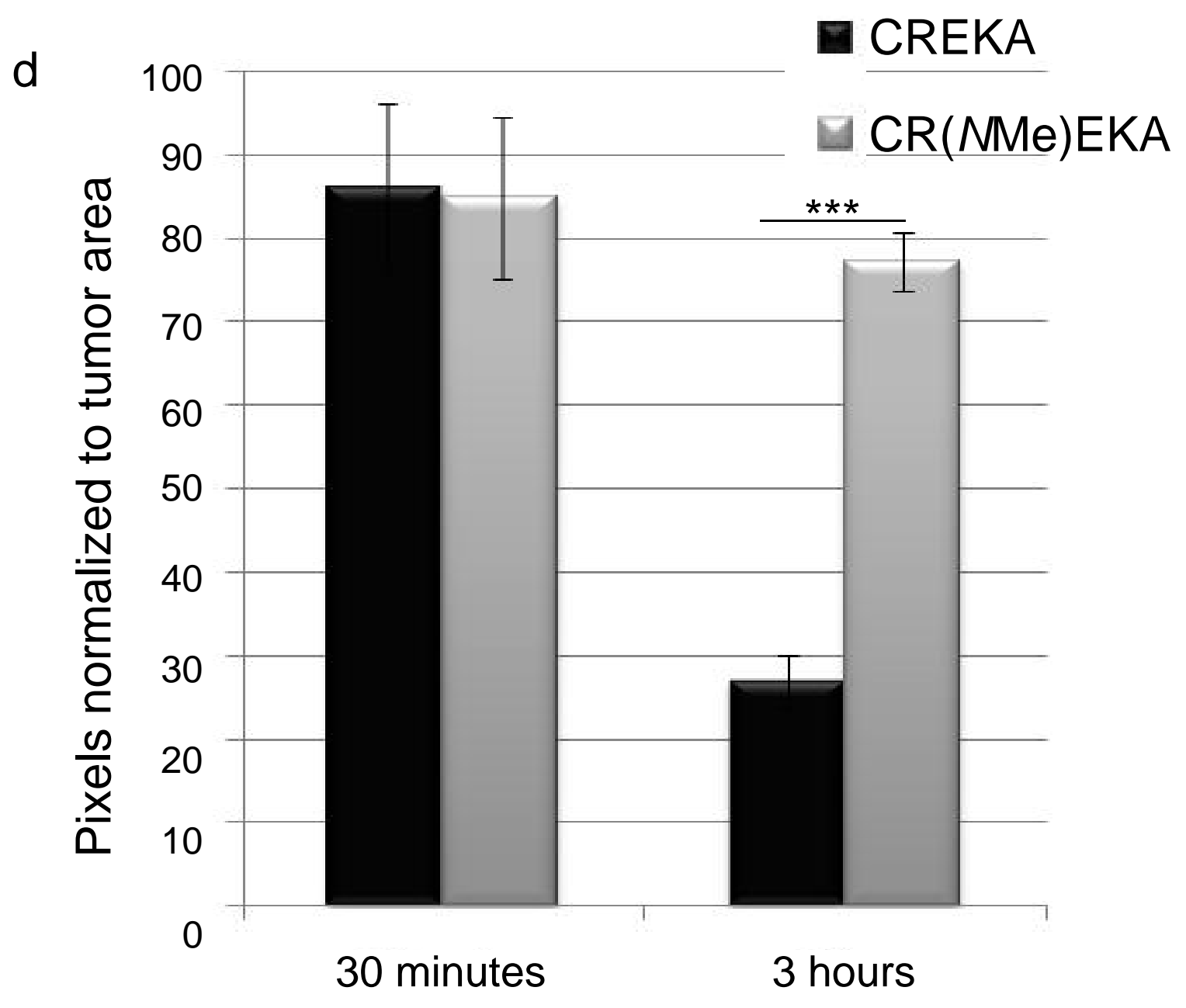


CRE(CMe)KA

Peptide

\section{CRE(CMe)KA -NW}

CRE(CMe)KA -NW

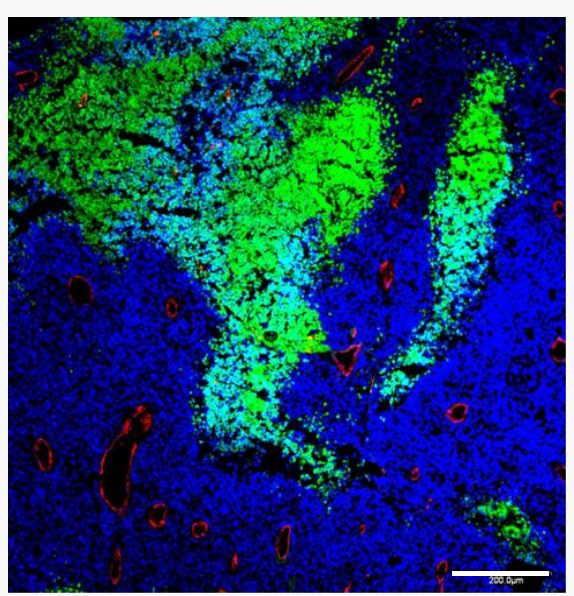

Fibrin(ogen)Tumor interior

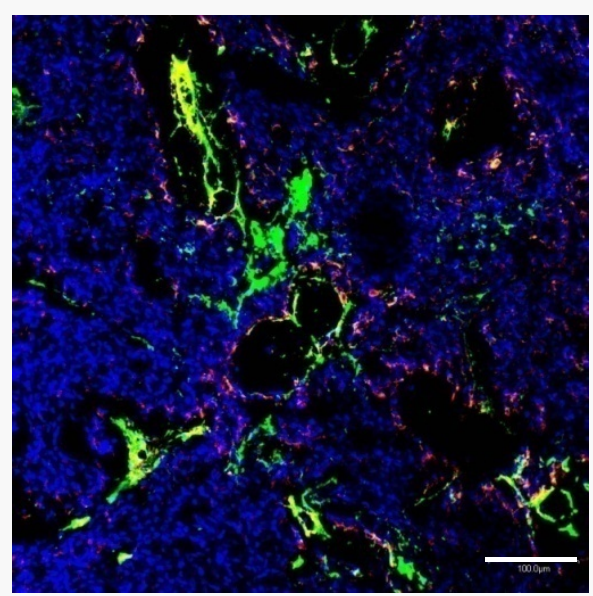

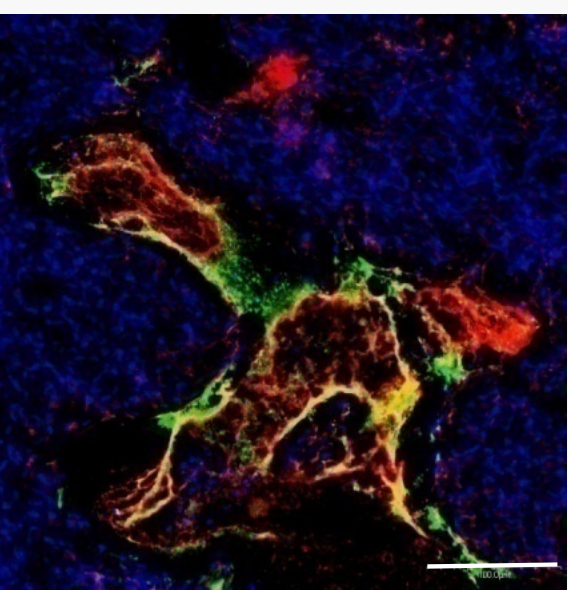

Fibrin(ogen)Tumor rim

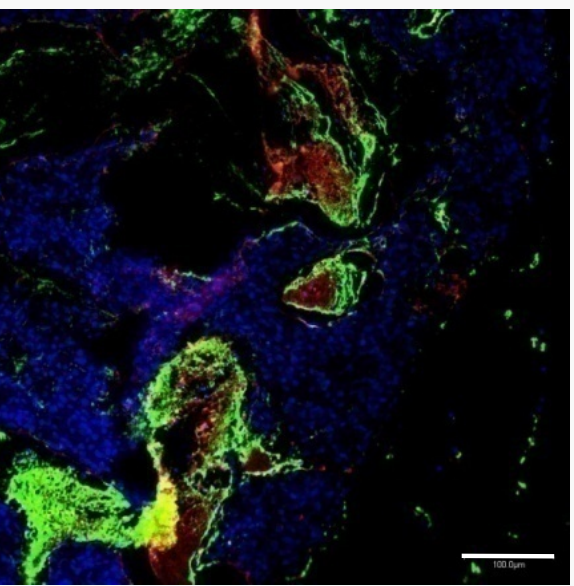




\section{0-274258 Agemy et al. Fig. 5}

a

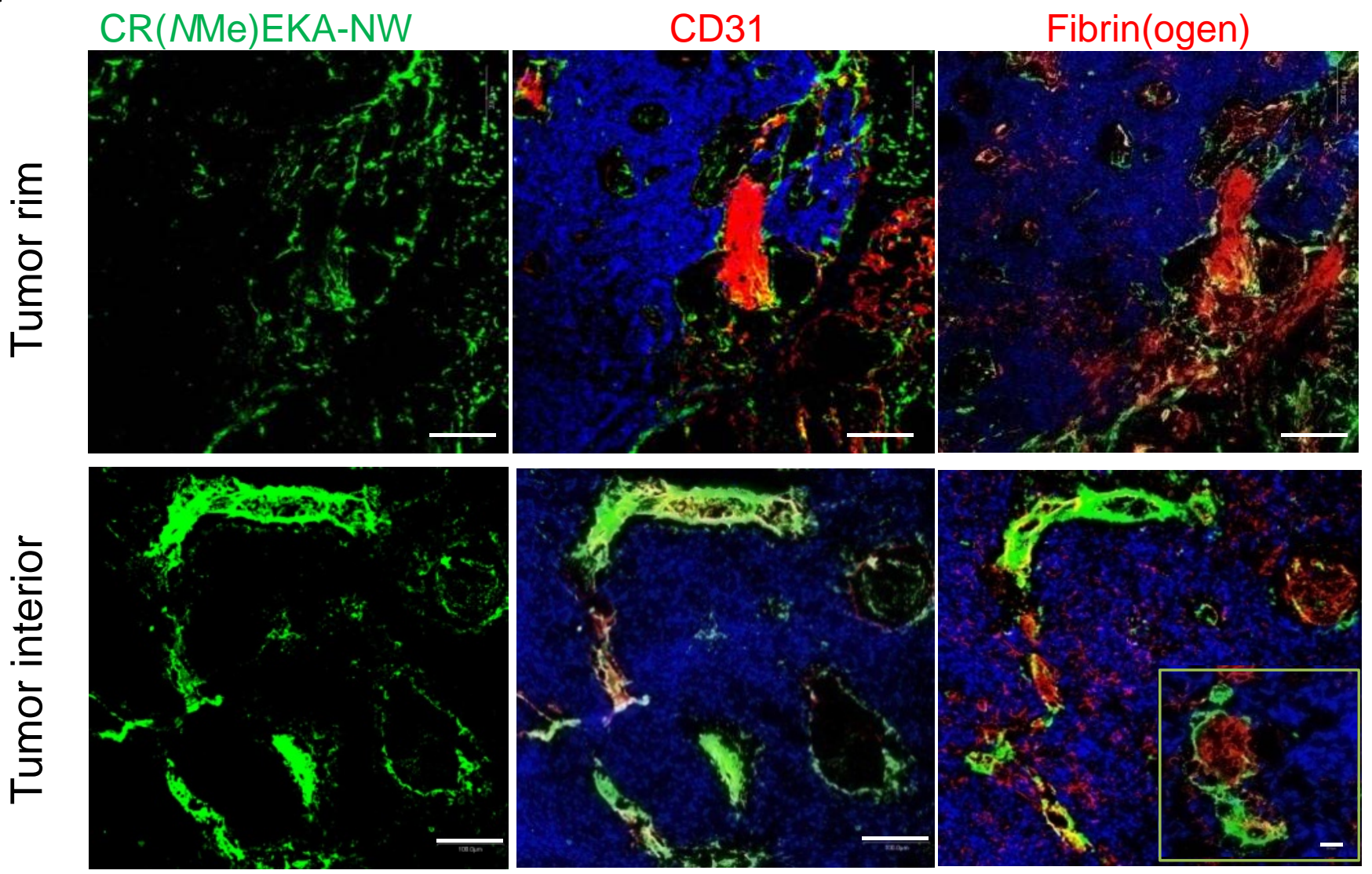

b

Pre-scan
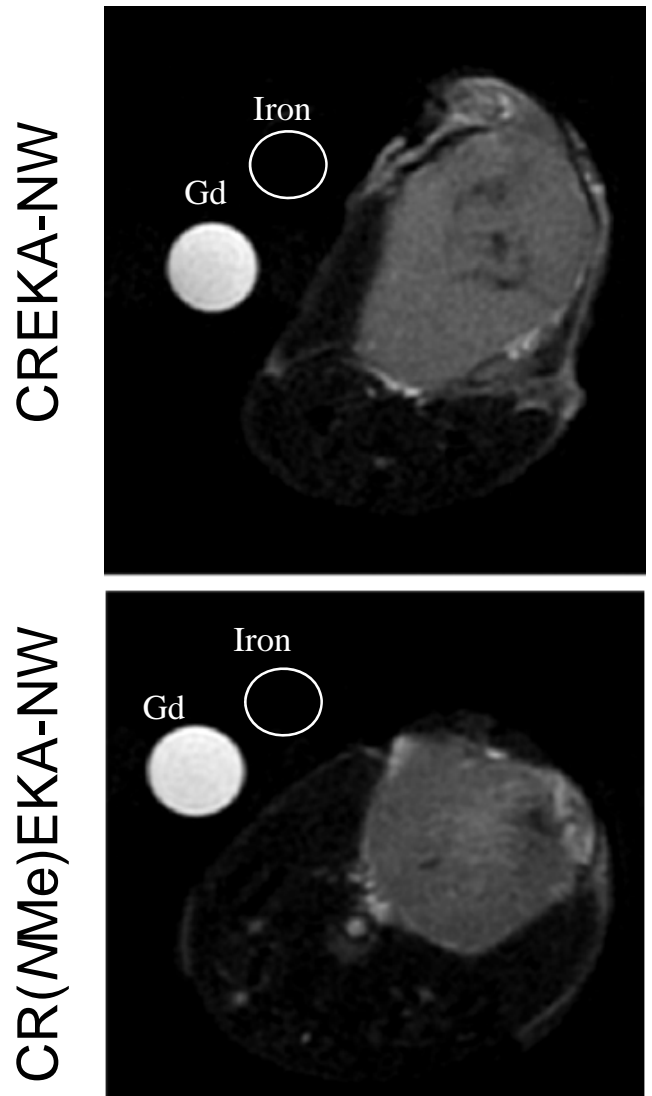

\section{7-8 hours post injection}
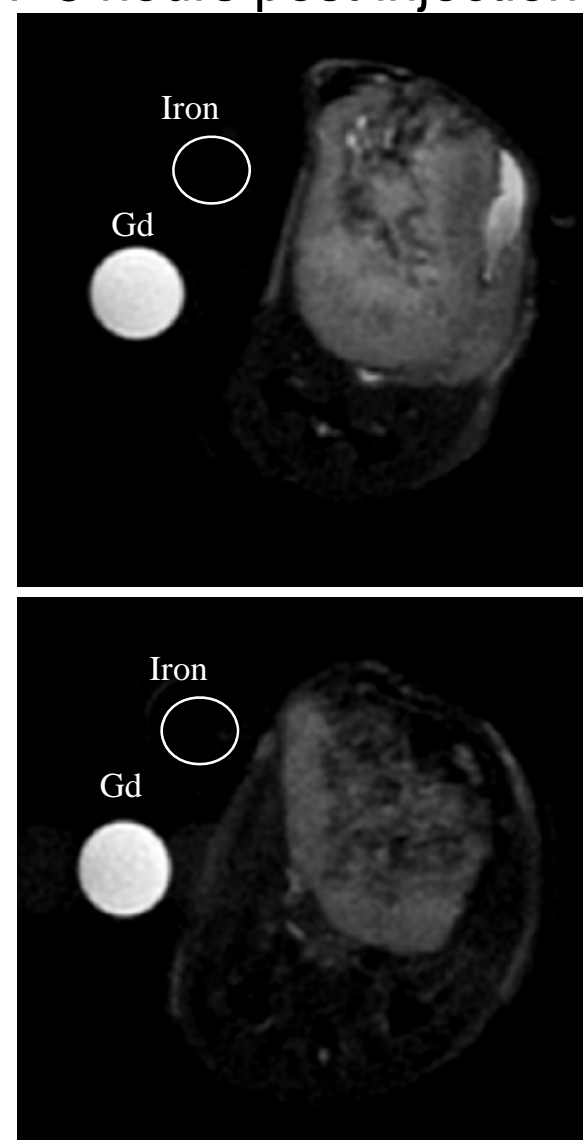
a

$$
\text { Peptide-PEG-NW }
$$

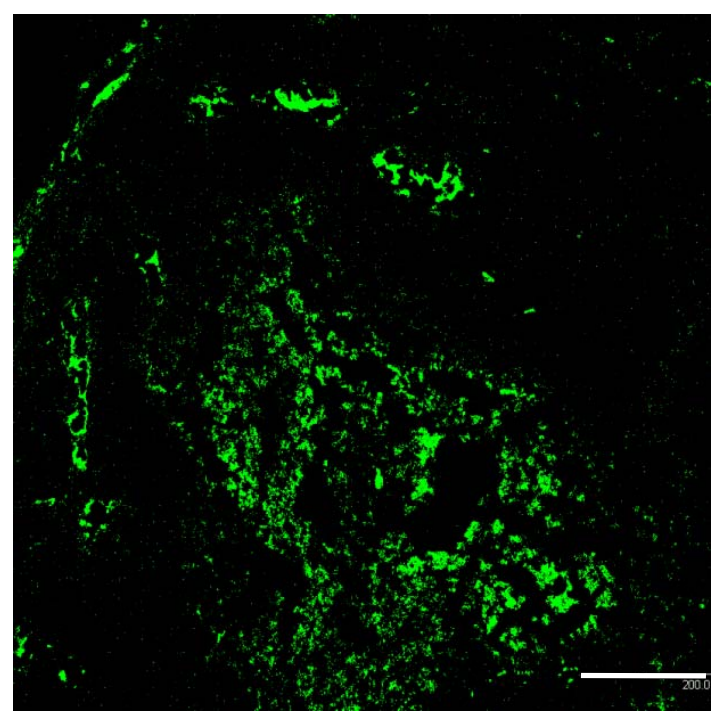

b

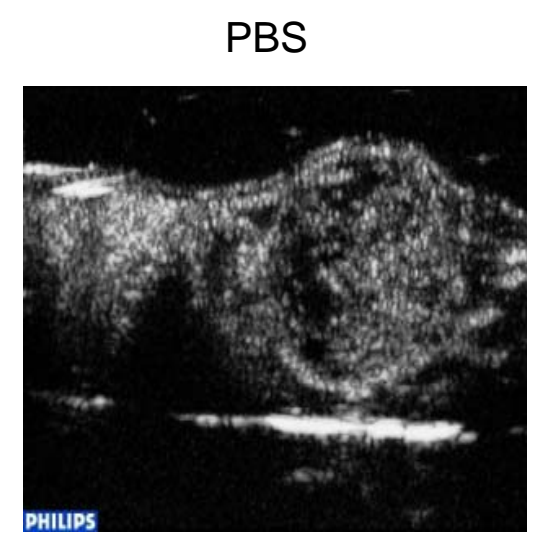

$$
\text { CR(NMe)EKA-PEG-NW }
$$

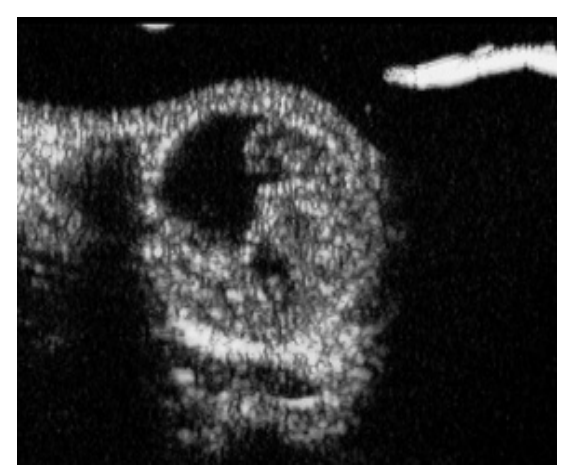

$86.1 \%$
$70 \%$
Peptide-PEG-NW+CD31+DAPI

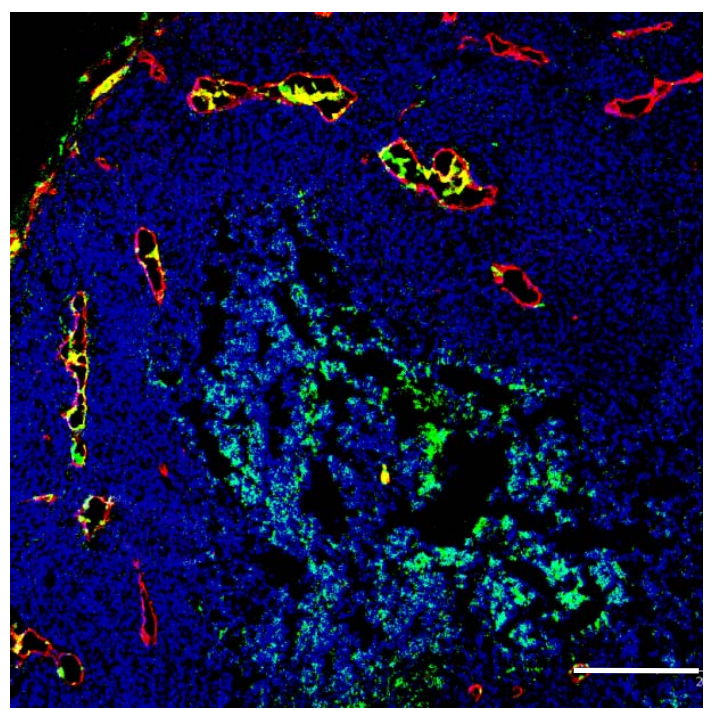

The percent area with enhancement
CRKDKC-NW+ CR(NMe)EKA-PEG-NW

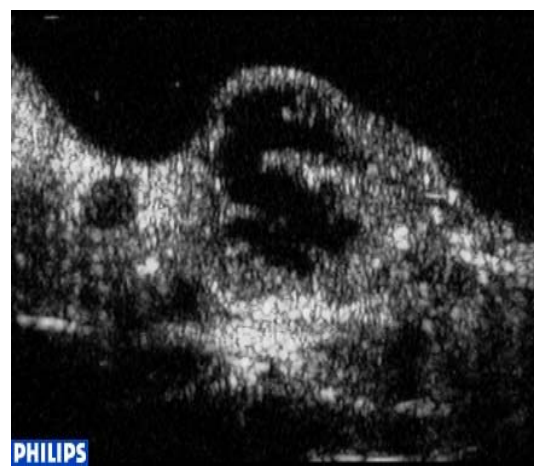

$30 \%$ 


\section{0-274258 Agemy et al. Fig. 6}

C

CRKDKC-PEG-NW+

CR(NMe)EKA-PEG-NW

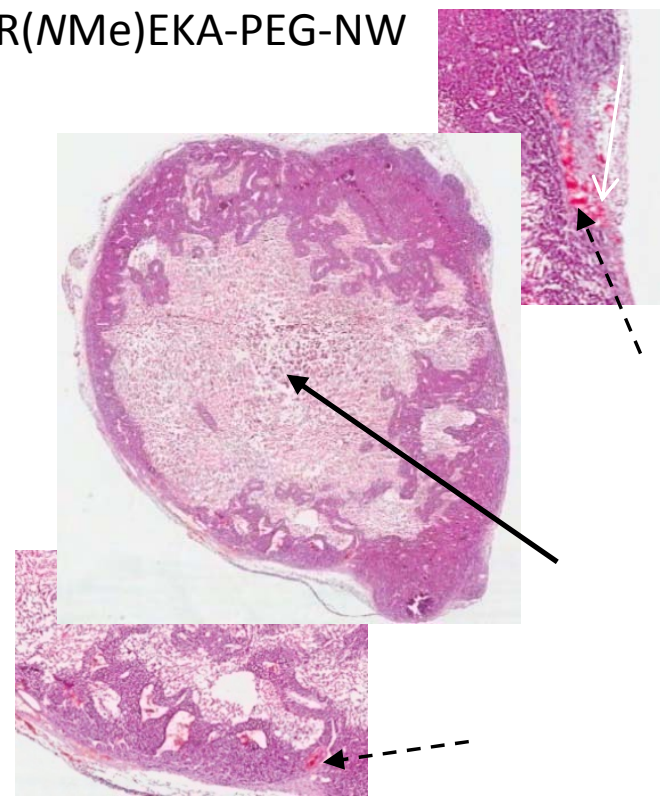

d

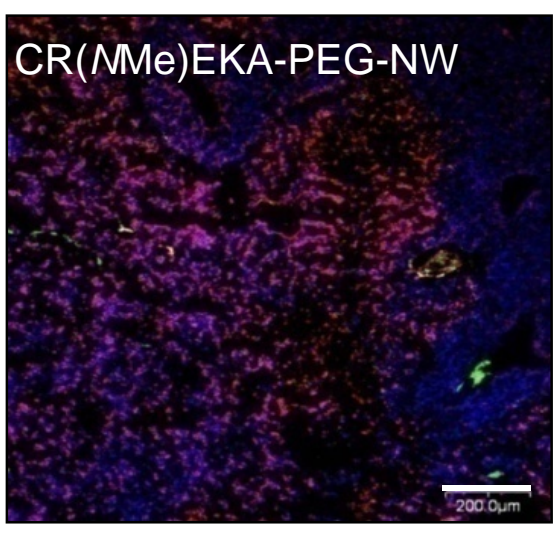

CR(NMe)EKA+CRKDKC -PEG-NW.
CRKDKC-PEG-NW

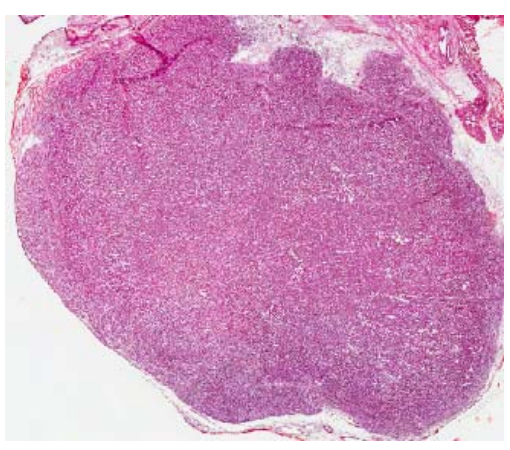

Control

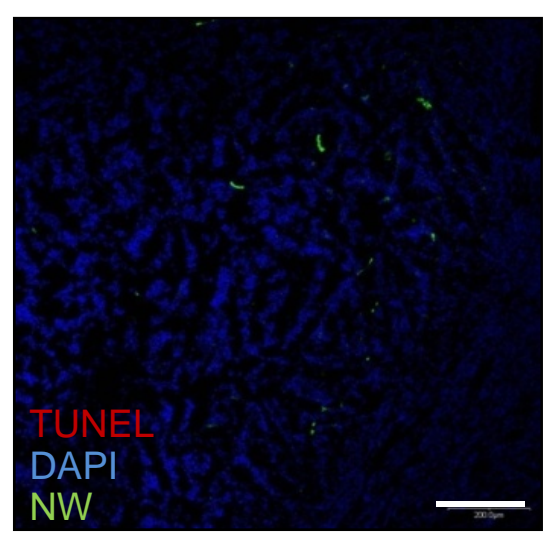

Control 


\section{0-274258 Agemy et al. Fig. 7}

a

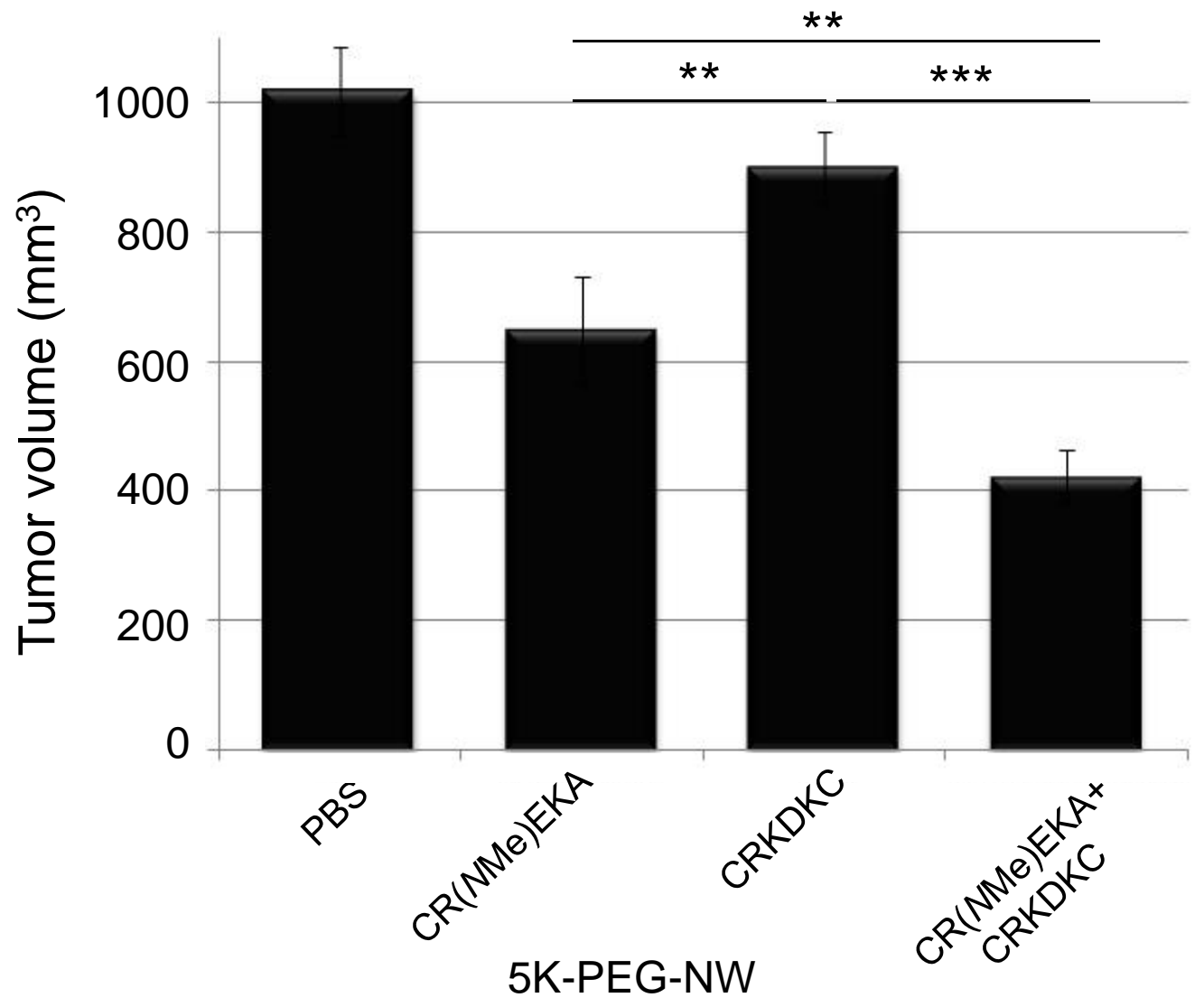

b

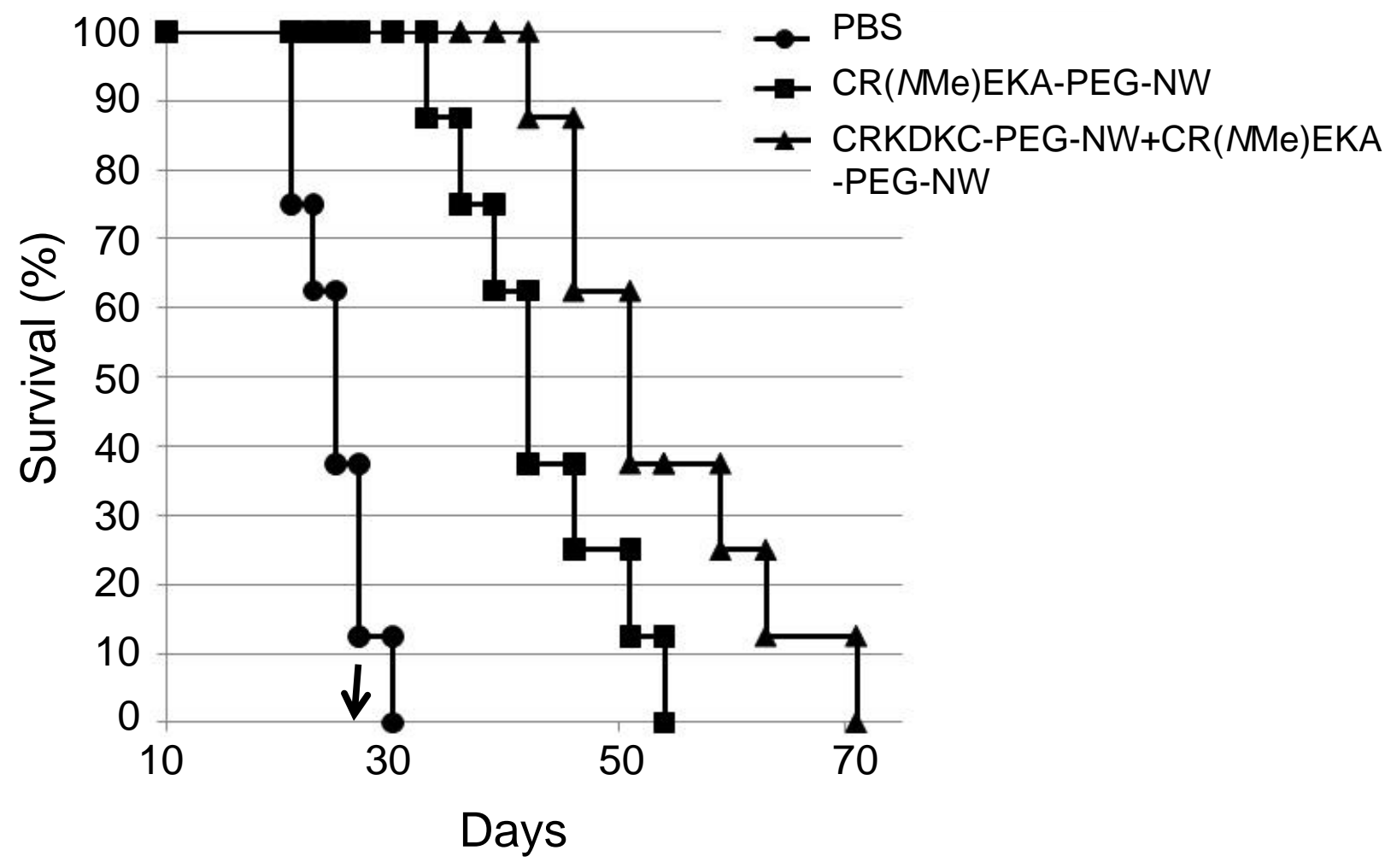

